1

2

3

4

5

6

7

8

9

10

11

12

13

14

15

16

17

18

19

20

21

22

23

24

\title{
Behavioral evidence for memory replay of video episodes in
} macaque monkeys

\author{
Shuzhen Zuo ${ }^{1, \square}$, Lei Wang ${ }^{1, \square}$, Junghan Shin $^{2}$, Yudian Cai ${ }^{1}$, Sang Wan Lee ${ }^{3}$, Kofi \\ Appiah $^{4}$, Yong-di Zhou ${ }^{5,7}$, Sze Chai Kwok ${ }^{1,6,7, *}$ \\ (1. \\ 1. Shanghai Key Laboratory of Brain Functional Genomics, Key Laboratory of Brain Functional Genomics \\ Ministry of Education, School of Psychology and Cognitive Science, East China Normal University, Shanghai, \\ China \\ 2. Program of Brain and Cognitive Engineering, Korea Advanced Institute of Science and Technology, Daejeon, \\ Republic of Korea \\ 3. Department of Bio and Brain Engineering, Korea Advanced Institute of Science and Technology, Daejeon, \\ Republic of Korea \\ 4. Sheffield Hallam University, Department of Computing, Sheffield, UK \\ 5. School of Psychology, Shenzhen University, Shenzhen, China \\ 6. Shanghai Key Laboratory of Magnetic Resonance, East China Normal University, Shanghai, China \\ 7. NYU-ECNU Institute of Brain and Cognitive Science at NYU Shanghai, Shanghai, China \\ $\square$ Joint-first authors. \\ * Lead contact and corresponding author. Email: sze-chai.kwok@st-hughs.oxon.org \\ Tel: +862120596020 \\ Fax: +862162233352
}




\section{ABSTRACT (148 WORDS)}

26 Humans recall the past by replaying fragments of events temporally. Here, we

27 demonstrate a similar effect in macaques. We trained six rhesus monkeys with a temporal-order judgement (TOJ) task and collected 5000 TOJ trials. In each trial, they watched a naturalistic video of about $10 \mathrm{~s}$ comprising two across-context clips, and after a 2-s delay, performed TOJ between two frames from the video. The monkeys apply a non-linear forward, time-compressed replay mechanism during the temporal-order judgement. In contrast with humans, such compression of replay is however not sophisticated enough to allow them to skip over irrelevant information by compressing the encoded video globally. We also reveal that the monkeys detect event contextual boundaries and such detection facilitates recall by an increased rate of information accumulation. Demonstration of a time-compressed, forward replay like pattern in the macaque monkeys provides insights into the evolution of episodic memory in our lineage.

Keywords: drift diffusion model framework; event boundary detection; forward replay-like pattern; naturalistic material; time compression of memory traces; temporal order judgement forward-replay mechanism during judgment of temporal-order between episodes. 


\section{INTRODUCTION}

Accumulating evidence indicate that non-human primates possess the ability to remember temporal relationships among events (1-3). The apes can remember the movies based on the temporal order of scenes (4) and keep track of past time of episodes (5), whereas macaque monkeys possess serial (6) and ordinal positions expertise for multi-item lists (7) and are able to categorize sequences of fractal images by their ordinal number (8). However, keeping track of and remembering the positional coding and forming associative chaining $(9,10)$ of lists of arbitrary items might diverge from how a semantically linked, temporally relational representation of real-life events is maintained and utilized.

In the human literature, it has been shown that episodes can be replayed sequentially based on learned structures (11), sensory information (12), and pictorial content (13). These findings suggest the possibility that monkeys can rely on a similar mechanism in recalling events that are linked temporally during temporal order judgement (TOJ). However, the extent to which mechanisms of temporal order judgement overlap across humans and monkeys remains undefined. One hypothesis is that the macaques can similarly rely on a scanning model for information retrieval akin to serial replay of episodes - to perform temporal order judgements $(2,3)$. By this account, following encoding streams of events, the animal performs retrieval through replaying the stream of information in a forward direction. In this way, retrieval time (RT) would be positively correlated to the temporal distance between the beginning of the stream and the target location. This account aligns with recent findings in rodents (14) and in humans of memory replay during cued-recall tasks across fragments of video episodes, showing characteristics of replay proceeding in a forward manner and being temporally compressed (12). Moreover, a more sophisticated feature of this mechanism is that memory replay is a fluidic process in which subjects are able to skip flexibly across sub-events (12). By this account, subjects can omit non-informative parts of episodes and replay a shorter episode (shorter than physical perception) in memory, which contains less information. This 
interpretation is supported by other works on mental simulation of paths (15) and video episodes (12). This latter account constitutes a global compression of parts of episodes (that allows skipping across sub-events) and is regarded as substantially superior to a strict forward replay mechanism.

In order to simulate dynamic flow of information as in real-life scenarios, we used naturalistic videos as experimental material to study the mechanism of memory retrieval of event order in the monkeys, which are more realistic than arbitrary items or images that were used in previous studies $(1,16)$. We used a temporal order judgement paradigm to examine whether and to what extent the pattern underlying memory retrieval conforms to a time-compressed, forward replay mechanism. In each trial, monkeys watched a naturalistic video, which is composed of two clips, and following a 2-s retention delay, made a temporal order judgement of choosing the frame that was shown earlier in the video between two frames extracted from that video (Figure 1A). The two frames were either extracted from the same clip or from two different clips of the video. Given that analyses on response latency can provide insights into the extent to which the monkeys' behavior might conform to the two putative replay models outlined above, we looked into the RT data. By applying representational similarity analyses (RSA), the Linear Approach to Threshold with Ergodic Rate (LATER) model and generalized linear models on the RT data, we examined the presence of replay-like behavioral patterns in the monkeys. Specifically, if monkeys recall the frames by their ordinal positions, this would imply a linear increase in their retrieval times. In contrast, if the memory search entails a complex processing of the content determined by their semantically linked, temporally relational linkage within the cinematic footage, we should observe evidence for some non-linear pattern.

Our results showed that macaque monkeys adopt a time-compressed, replay-like pattern to search within representation of continuous information. In addition, by comparing between human and macaque data, we adjudicated between the two aforementioned aspects of the replay models. The results showed a discrepancy between the two species that the monkeys do not compress the cinematic events 
106 globally as effectively as in humans. Finally, we revealed that the monkeys can make

107 use of context changes to facilitate the memory retrieval by an increased rate of

108 information accumulation in a drift diffusion model framework.

\section{RESULTS}

111

112

113

114

115

116

117

118

\section{Human-like forward replay in macaques}

All six monkeys learned to perform the temporal order judgement task with dynamic cinematic videos as encoded content (Figure 1B left and Movie S1). Note that there are two main kinds of TOJ trials: "within-context" and "across-context" trials (Figure 1A). Here, we will be first concerned with the response times (RT) data from "within-context" trials to examine TOJ mechanisms, while RT data from "across-context" trials will be used to test for effects by context changes (event boundary) in subsequent subsections.

We first addressed our main hypothesis by examining changes in RT using only within-context trials. We regressed RT as a function of temporal similarity (TS) for within-context trials and found a significant negative relationship between TS and RT, all $P<0.001$ (one monkey with $P=0.02$, Table $\mathbf{S 2}$ upper panel and Figure S1). The results were replicated when considering only correct trials or only incorrect trials separately (Table $\mathbf{S} 2$ middle/bottom panels). This suggests that the monkeys are systemically faster in identifying frames that are located earlier in the video. One possibility is that the monkeys perform TOJ relying on some form of memory replay by recalling the events coded at specific ordinal positions in a serial manner. To test this hypothesis, in each trial we explicitly looked at the relationship between RT and the location of the frame within the video which the monkeys have chosen ("chosen frame location", as indexed by the ordinal frame numbers in the video). By correlating RT as a function of chosen frame location, we indeed found a significantly positive relationship between RT and chosen frame location in all monkeys, all $P<$ 0.001 (two monkeys with $P=0.002$, Figure $1 \mathrm{C}$ and Table 1 top panel). The same pattern was replicated when only correct trials (Table 1 middle panel) or incorrect 
trials (Table 1 bottom panel) were included for analysis, suggesting that the putative retrieval RT patterns are not affected by the memory outcome. This pattern of result is also replicated using logarithmically transformed RT data, all $P<0.001$. After taking individual variability into consideration, we obtained the same result with reciprocal latency as a function of chosen frame location for the average of all monkeys (Figure 2A). While the result suggests that the monkeys' judgments are faster to respond to probe frames that are located at the earlier parts of the videos, it also indicates that the search does not follow a linear function. This non-linear relationship is important because it rules out alternative explanations that the main effects are simply resultant from positional effects. Rather, the non-linear change in slope indicates there are other factors in play that the monkeys might group (or parse) the content according to the relational storyline structure, and do not merely recall the frames/items as of their ordinal positions in a fixed linear manner.

To address the direction and speed of memory reply, reaction times at retrieval were compared between TOJ frames that are within Clip 1 versus Clip 2. During TOJ retrieval, frames that were presented in Clip 1 (mean reaction time $\square=\square 1.59 \mathrm{~s}$ ) $\square$ were retrieved significantly faster than the frames that were experienced in Clip 2 (mean reaction time $\square=\square 1.88 \mathrm{~s}$ ) (one-tailed $t 5=-4.533 ; P=0.003$; Cohen's $\mathrm{d} \square=-0.54 ; 95 \%$ CI: [-inf, -0.158]; $\log (\mathrm{RT})$ : one-tailed $t 5 \square=\square-6.473 ; P \square=6.558 \times \mathrm{e}^{-4}$; Cohen's $\mathrm{d} \square=\square-0.69 ; 95 \% \mathrm{CI}$ : [-inf, -0.114]). These findings again confirm that the replay of the video takes place in a forward direction.

Moreover, since the latency required to responding to the chosen frames is much smaller than the duration of the videos themselves, the replay of the video must have been conducted at a compressed speed (i.e., memory replay was faster relative to perception during video-watching). The difference in reaction time between the very first frame and the last frame was averaged at $942 \mathrm{~ms}$ (range: $468-1859 \mathrm{~ms}$ ). This is equivalent to $94.2 \mathrm{~ms}$ to scan through per second of the video and corresponds to a compression factor of 10.61 during replay in these monkeys (compression factor for each monkey: Jupiter $=13.59$, Mars $=7.39$, Saturn $=14.80$, Mercury $=21.37$, Uranus $=$ 17.78, Neptune $=5.38$, see Figure $1 \mathrm{C}$ ). This is comparable to a compression factor of 
16513.7 observed in humans, corroborating the notion of forward replay and those 166 findings in humans (12).

A

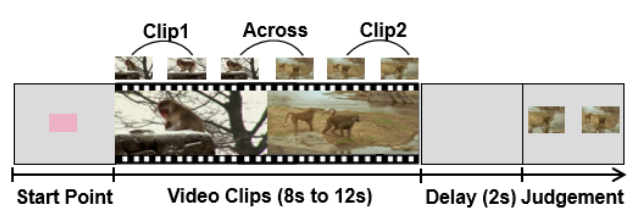

C

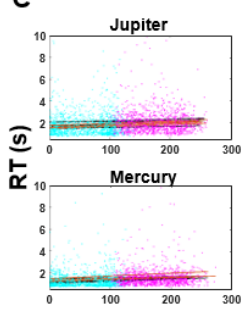

D
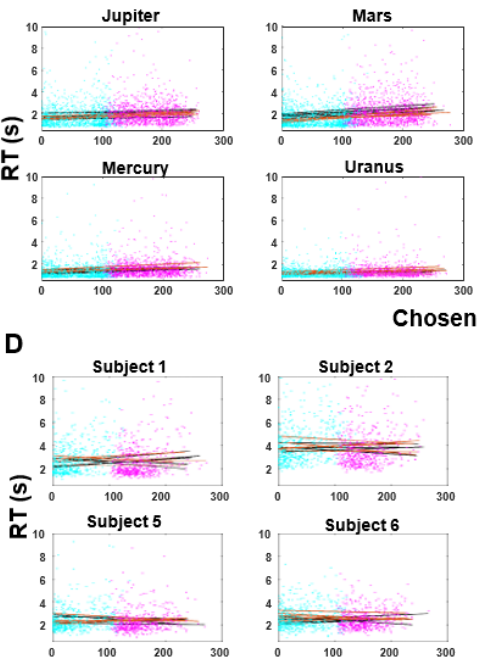

B

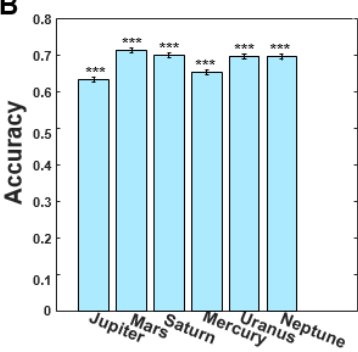

Chosen frame location

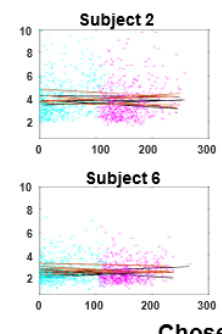

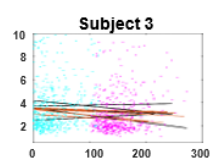
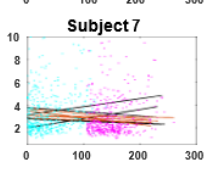
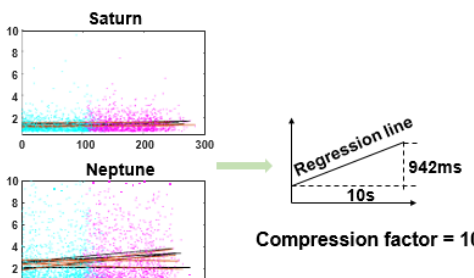

Compression factor $=10.61$

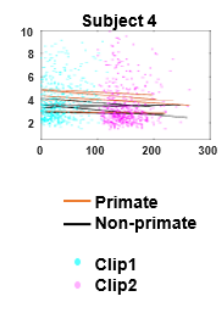

167

Figure 1. TOJ task schema and RT results. (A) On each trial, the monkey watched a video (8-12s, comprising two 4-6s video clips), and following a 2-s retention delay, made temporal order judgement between two probe frames extracted from the video. The monkeys were required to choose the frame that was presented earlier in the video for water reward. (B) Task performance of six monkeys. Proportion correct for the six monkeys (left); mean reaction times for three trial types (right). Error bars are standard error of the mean $(\mathrm{SEM})$. $* * *$ denotes $\mathrm{P}<0.001$. (C) Linear regression analysis of reaction time (RT) as a function of chosen frame location, see also Table 1. (D) Linear regression analysis of RT as a function of chosen frame location for each human participant, see also Table S3. In (C) and (D), black lines and orange lines refer to lists of non-primate and primate videos respectively (with five repetitions collapsed for monkeys and two repetitions collapsed for human participants). All 
180

181

182

183

184

185

186

187

188

189

190

191

192

193

194

195

196

197

198

199

200

201

202

203

204

205 responses in within-context are shown, with cyan and magenta dots denoting whether the chosen probe frames were extracted from either Clip 1 or Clip 2 respectively.

We also ran the same sets of analyses on human participant data for comparison between the two species. Showing a completely opposite pattern, regression analyses on human subjects showed a positive relationship between temporal similarity (TS) and RT for all participants, all $P<0.01$ (Table S3 upper panel). These results imply that the more similar the two frames to be judged, the longer time needed for retrieve temporal order information. There was also no observable RT/ chosen frame location slope in the human data (if anything, it shows an opposite trend; see Figure 1D \& Table S3 bottom panel). In sum, we found that our monkeys might have performed TOJ of video episodes using a forward search of ordered elements in the mnemonic representation at the time of memory test with a non-linear compression function. By contrasting with Figure $\mathbf{2 A}$, it is notable that when reciprocal latency as a function of chosen frame location is analyzed in the humans, as shown in Figure 2B, a very different pattern emerges, suggesting some form of mechanistic discrepancy between the species. We will examine these aspects in detail in the next section.

We have also run a sliding-window average analysis to illustrate how accuracy varies as a function of the target frames location (Figure 2C for monkeys; Figure 2D for humans). It is interesting that in both species there is a mild trend that their proportion correct show a little blip right after the beginning of Clip 2. This characteristic might be related to their ability in detecting the boundaries (cf. results and discussion related to Figure 6). However, given that we are primarily concerned with using response times as quantitative measures to examine the TOJ mechanisms, we do not speculate further into this aspect. 

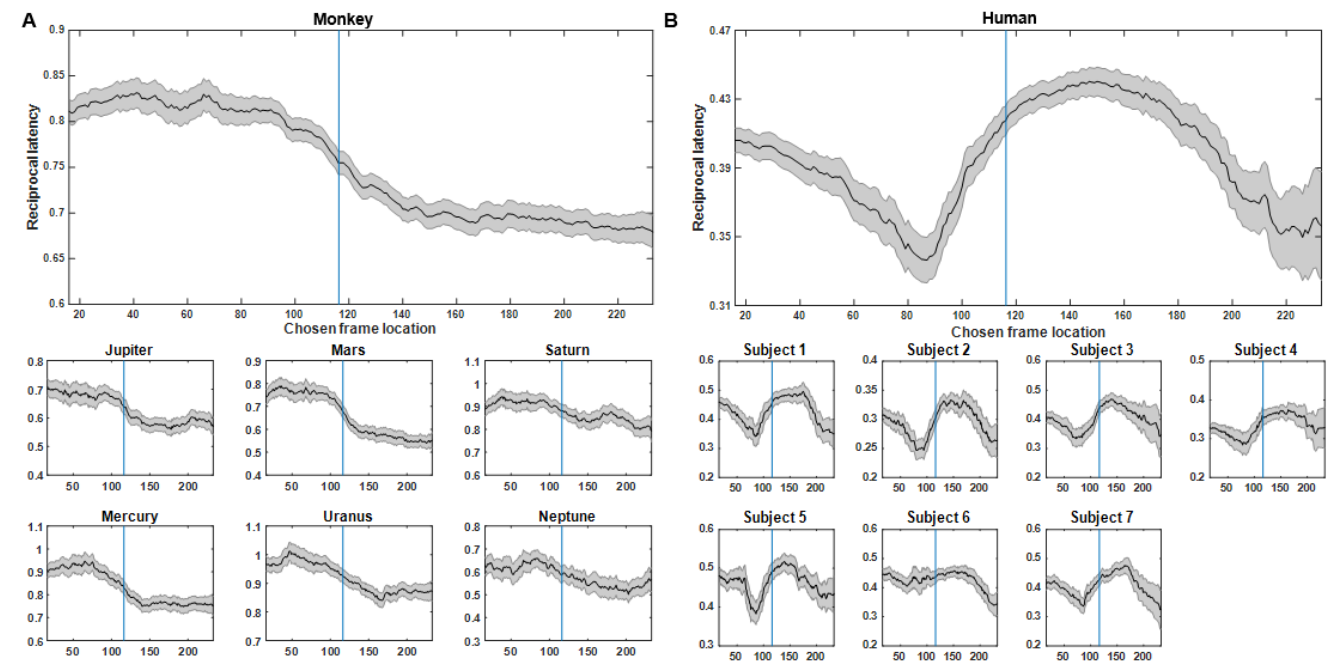

C
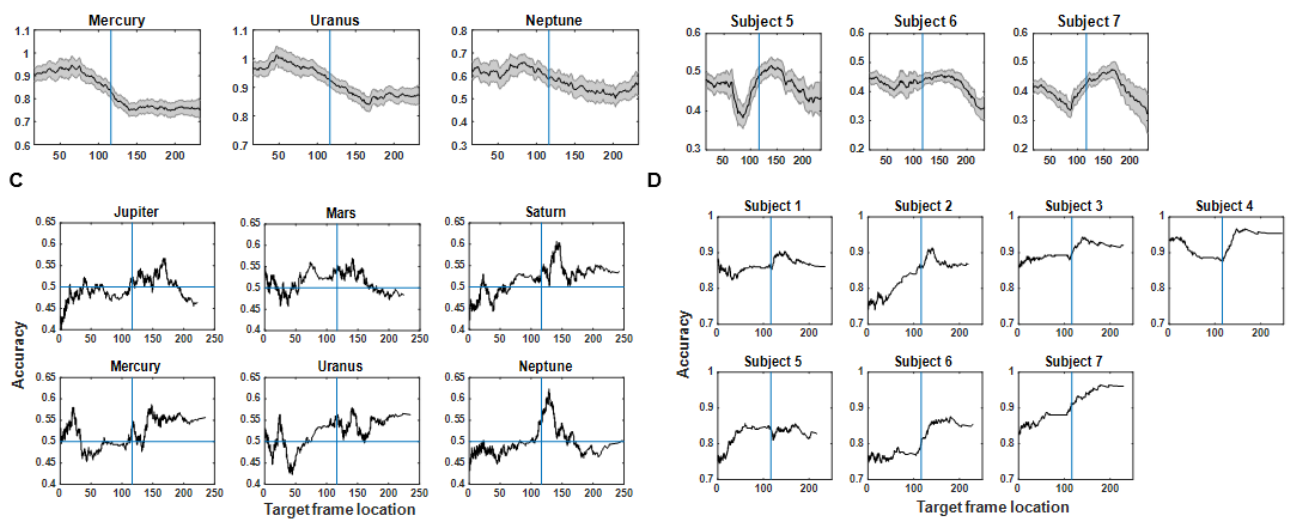

207

Figure 2. Moving average analysis based on reciprocal latency and accuracy for

208

209

210

211

212

213

214

215

216

217

218

219

220

221

monkeys (left panels) and human participants (right panels). (A) Reciprocal latency for monkeys as a function of chosen frame location for the average of all animals (upper panel) in within-context condition, with results of six individual monkey shown (lower panel). The relationship between chosen frame location and RT follows a non-linear pattern. (B) Reciprocal latency for human participants as a function of chosen frame location for the average of all human subjects (upper panel) in within-context condition, with results of individual subject shown (lower panel). In (A) and (B), shaded region denotes confidence intervals; the blue line denotes the mean boundary location between Clip 1 and Clip 2 across all trials $\left(116^{\text {th }}\right.$ frame). (C) Individual monkey's proportion correct as a function of target frame location in within-context condition. Horizontal blue lines denote chance level accuracy. (D) Individual human subject's proportion correct as a function of target frame location in within-context condition. 
222

223

224

225

226

227

228

229

230

231

232

233

234

235

236

237

238

Table 1. One sample t-tests results of the slopes of RT as a function of chosen frame location for each monkey. The three panels correspond to analyses performed using all trials (top), only correct trials (middle), and only incorrect trials (bottom). Same slope patterns were observed irrespective of correctness.

\begin{tabular}{|c|c|c|c|c|c|c|c|}
\hline \multirow{2}{*}{ Monkeys } & \multirow{2}{*}{ Mean (SEM) } & \multirow{2}{*}{ t-statistics } & \multirow{2}{*}{ d.f. } & \multirow{2}{*}{$p$-value } & \multirow{2}{*}{ Cohen's d } & \multicolumn{2}{|c|}{$95 \%$ confidence interval } \\
\hline & & & & & & Lower & Upper \\
\hline \multicolumn{8}{|c|}{ Slope of reaction times/chosen frame location tested against zero (all trials) } \\
\hline Jupiter & $0.0015(0.0017)$ & 6.06 & 49 & $<0.001$ & 0.86 & 0.0010 & 0.0020 \\
\hline Mars & $0.0031(0.0027)$ & 7.93 & 49 & $<0.001$ & 1.12 & 0.0023 & 0.0038 \\
\hline Saturn & $0.0007(0.0016)$ & 3.29 & 49 & 0.002 & 0.47 & 0.0003 & 0.0012 \\
\hline Mercury & $0.0017(0.0018)$ & 6.77 & 49 & $<0.001$ & 0.96 & 0.0012 & 0.0022 \\
\hline Uranus & $0.0011(0.0014)$ & 5.12 & 49 & $<0.001$ & 0.72 & 0.0006 & 0.0015 \\
\hline Neptune & $0.0024(0.0049)$ & 3.34 & 46 & 0.002 & 0.49 & 0.0009 & 0.0038 \\
\hline \multicolumn{8}{|c|}{ Slope of reaction times/chosen frame location tested against zero (correct trials) } \\
\hline Jupiter & $0.0023(0.0042)$ & 3.85 & 49 & $<0.001$ & 0.55 & 0.0011 & 0.0035 \\
\hline Mars & $0.0029(0.0034)$ & 5.98 & 49 & $<0.001$ & 0.85 & 0.0019 & 0.0038 \\
\hline Saturn & $0.0009(0.0021)$ & 3.03 & 49 & 0.004 & 0.43 & 0.0003 & 0.0015 \\
\hline Mercury & $0.0018(0.0026)$ & 4.79 & 49 & $<0.001$ & 0.68 & 0.0010 & 0.0025 \\
\hline Uranus & $0.0012(0.0021)$ & 4.01 & 49 & $<0.001$ & 0.57 & 0.0006 & 0.0018 \\
\hline Neptune & $0.0030(0.0070)$ & 2.94 & 46 & 0.005 & 0.43 & 0.0009 & 0.0051 \\
\hline \multicolumn{8}{|c|}{ Slope of reaction times/chosen frame location tested against zero (Incorrect trials) } \\
\hline Jupiter & $0.0016(0.0032)$ & 3.49 & 49 & 0.001 & 0.49 & 0.0007 & 0.0025 \\
\hline Mars & $0.0044(0.0039)$ & 8.00 & 49 & $<0.001$ & 1.13 & 0.0033 & 0.0055 \\
\hline Saturn & $0.0011(0.0025)$ & 3.20 & 49 & 0.002 & 0.45 & 0.0004 & 0.0018 \\
\hline Mercury & $0.0022(0.0026)$ & 5.96 & 49 & $<0.001$ & 0.84 & 0.0014 & 0.0029 \\
\hline Uranus & $0.0012(0.0019)$ & 4.52 & 49 & $<0.001$ & 0.64 & 0.0007 & 0.0017 \\
\hline Neptune & $0.0034(0.0075)$ & 3.15 & 46 & 0.003 & 0.46 & 0.0012 & 0.0056 \\
\hline
\end{tabular}

\section{Discrepancy with humans: Compression of replay is local but not global}

It has been shown in the humans that memory replay is not a straightforward recapitulation of the original experience. Subjects can skip through their memories on a faster time scale across segments of a video episode than within-segment by skipping flexibly over salient elements such as video boundaries within episodes (12). We propose two possible models with respect to whether the compression is global or not over the whole video. If there is a global compression of the video during replay, the speed to initiate replay for Clip 2 would be sooner than the endpoint of replay for Clip 1 due to the animal being able to skip over the entire Clip 1 to the beginning of Clip 2 (Global-compression model, Figure 3A right panel). However, if the monkeys are not equipped with the ability of skipping video 
239

240

241

242

243

244

245

246

247

248

249

250

251

252

253

254

255

256

257

258

259

260

261

262

263

segments during the replay process, we would expect a linear increase of retrieval time with chosen frame location irrespective of the boundary (Strict forward model, Figure 3A left). We tested statistically whether the speed to initiate replay for Clip 2 slowed down compared to that for Clip 1. We divided each video into eight equal segments and derived cross-correlations computed on pairs of averaged condition-wise RTs based on chosen frame locations using a representational similarity analysis. The RT for TOJ between each segment of the video increases linearly according to their position in encoding (Figure 3B left). We tested these against a hypothetical "Strict forward" model and found significant correlation with the Strict forward model $(r=0.66, P=0.009)$, but not with the Global compression model $(r=-0.16, P=0.802)$ (Figure 3B right). These statistics also remain significant for the Strict forward model when we divided the video into either $10(P=0.030)$ or 14 equal segments $(P=0.020)$. The same patterns are also obtained when considering correct trials (Strict forward model: $r=0.37, P=0.040$; Global compression model: $r$ $=-0.02, P=0.545$ ) or incorrect trials (Strict forward model: $r=0.45, P=0.010$; Global compression model: $r=-0.06, P=0.545)$ separately. Contrarily, these correlational patterns with the Strict forward model are not observed in the human subjects $(r=-0.11, P=0.703)$, but rather we observe a trend favoring the global compression model instead ( $r=0.39, P=0.069)$ (Figure 3B right).

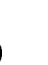


264
A

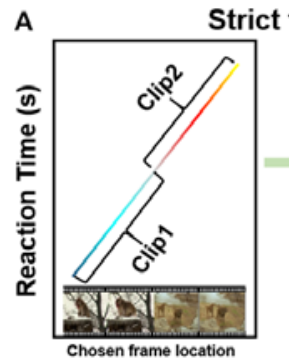

B
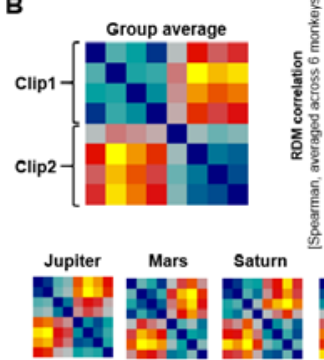
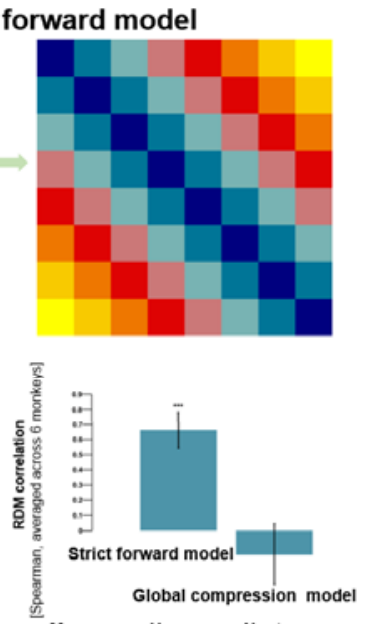

Mercury Uranus Neptune
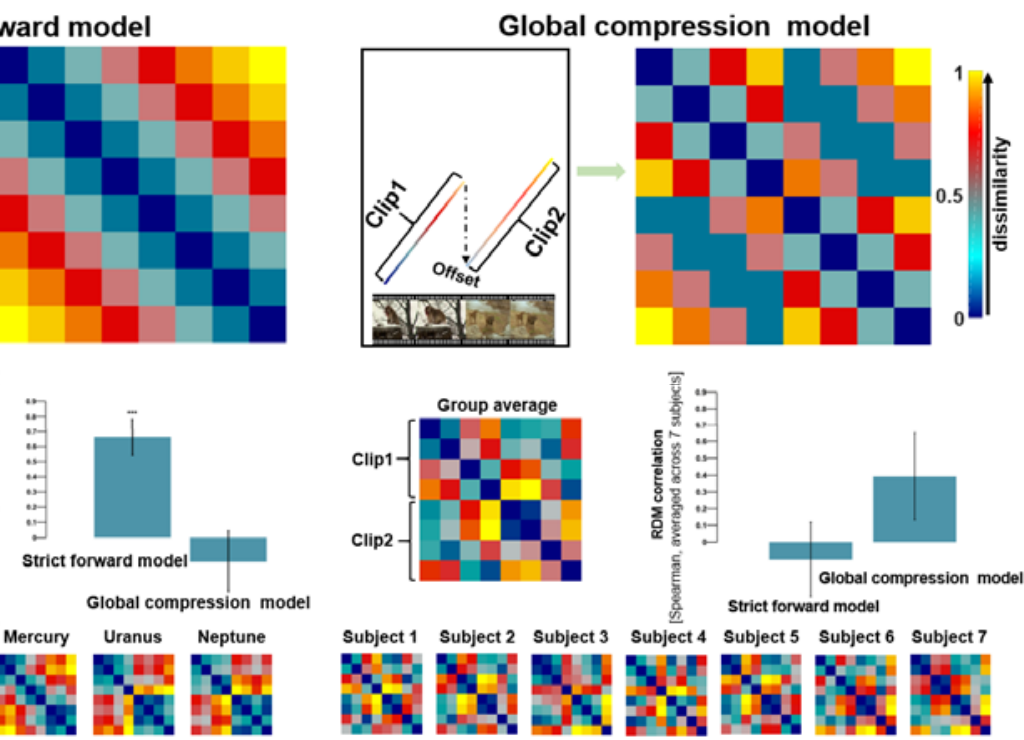

Figure 3. Model comparison using representative similarity analysis. (A) Visualization of two candidate models as RDMs. Pattern of reaction time (rank-transformed values) as a function of increasing chosen frame location underlying the two hypothetical models. The colors of Clip 1 and Clip 2 evolve increasingly with the temporal progression of the video (left); and their respective hypothetical RDMs (right). The reduction in RT indicated by an arrow between Clip 1 and Clip 2 is defined as "offset"; the magnitude of such "offset" is arbitrary but see further analysis in Figure 4. (B) We segmented the videos into eight equal segments and the RDMs contain pairwise Euclidean distances between these different segments for the group average (Monkeys: left; humans: right) and each individual separately (Monkeys: left bottom; humans: right bottom). RDM correlation tests between behavioral RDMs and two candidate RDMs show that the monkeys replay the footage using a Strict forward strategy and show little evidence for the Global compression strategy. Humans show an opposite pattern from the macaques. In the humans (right panels), the Global compression model shows a higher correlation with behavioral RDM (marginally insignificant $r=0.39, P=0.069$ ) than with the Strict forward model $(r=-0.11, P=0.703)$. Error bars indicate the standard error of the mean based on 100 iterations of randomization. $P$ values are FDR-corrected $(* * *$ denotes $P<0.001)$. 


\section{Factors modulating the model: "offsets" for search and memory-search RT slope}

We defined the reduced RT to initiate replay for Clip 2 as "offsets" in initiating search in Clip 2 by skipping the non-informative Clip 1 (Figure 3A). With respect to the detailed differences between the two models, one may wonder whether and how the "offsets" between Clip 1 and Clip 2 might influence the results. Especially for the Global compression model, changes of this parameter will cause changes in the RDMs. To address this concern, we simulated an array of RDMs by systemically varying the offset parameter and produced 11 hypothetical models ranging from an absolute Global compression model (model 1, most left in Figure 4A), to a Strict-forward model (model 6, middle in Figure 4A), and beyond $\left(7^{\text {th }}\right.$ to $11^{\text {th }}$ models, right in Figure 4A). We then tested each individual monkeys' data with each of these 11 models. The results show that the Spearman correlation values between the monkey's data and hypothetical RDMs reach an asymptote of around $r=0.8$ as the offset parameter tend to zero, and notably, the correlation values only improve minimally with increasing offsets (Figure 4B, with individual's RT RDM displayed as insets). These suggest that the monkeys have processed the video as a holistic chunk of information rather than taking advantage of skipping the non-informative first clip when the two probe frames were in Clip 2. For comparison, we also tested human participant's data against each of these 11 hypothetical models and found a completely opposite pattern in the humans (Figure 4C, with individual's RT RDM displayed as insets). Taken together, we reveal a discrepancy between human and macaque performance in terms of their ability to compress past (irrelevant) information during TOJ.

.


bioRxiv preprint doi: https://doi org/10.1101/2020.01.10.902130; this version posted January 11 2020. The copyright holder for this preprint (which was not certified by peer review) is the author/funder, who has granted bioRxiv a license to display the preprint in perpetuity. It is made available under aCC-BY 4.0 International license.

A
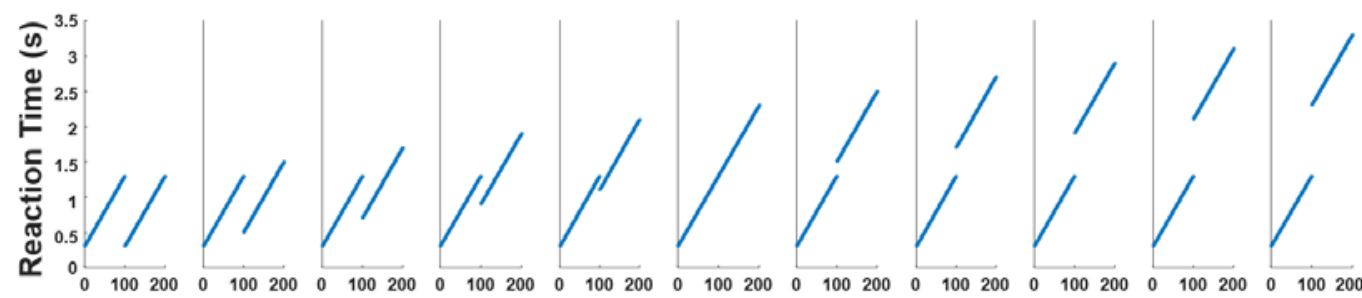

Chosen frame location

312
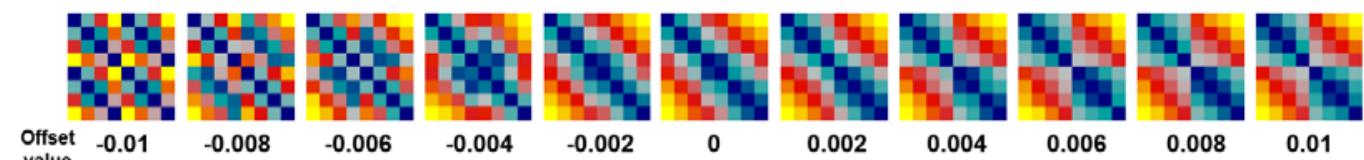

B
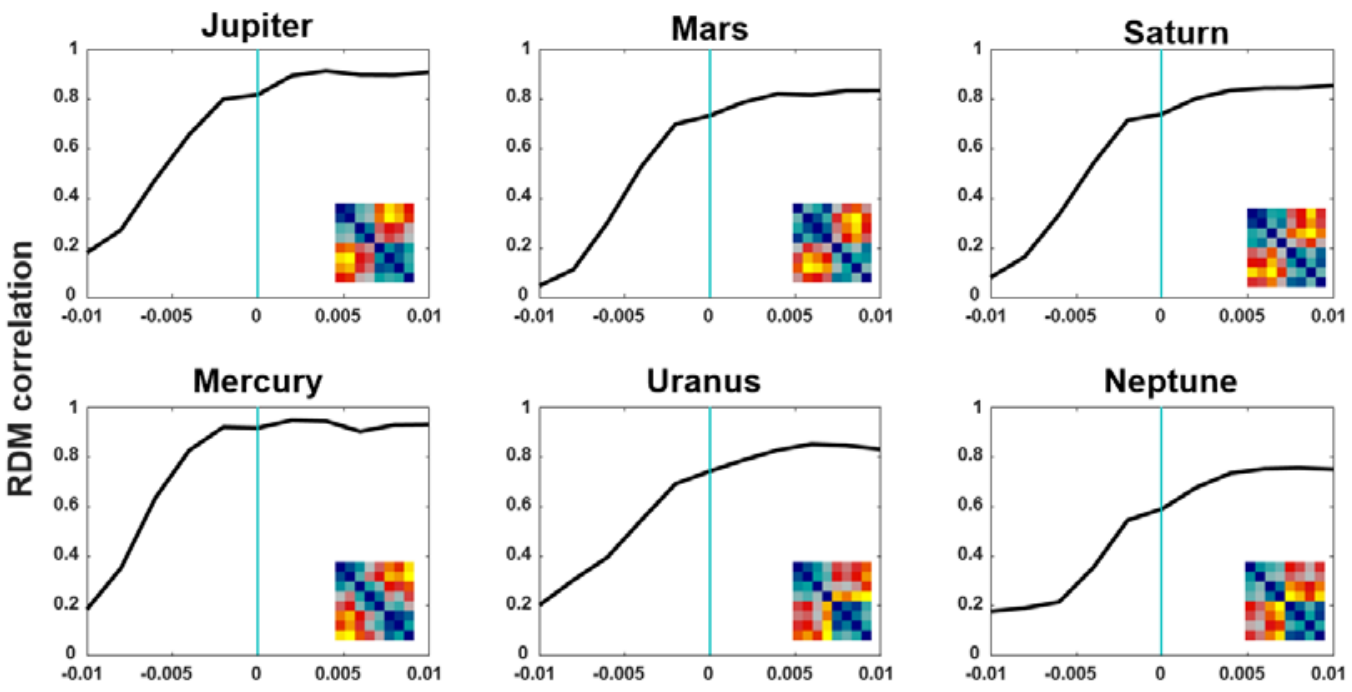

313
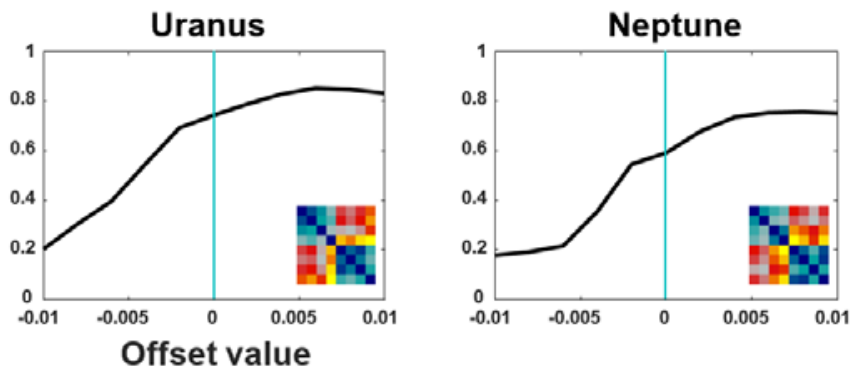

C
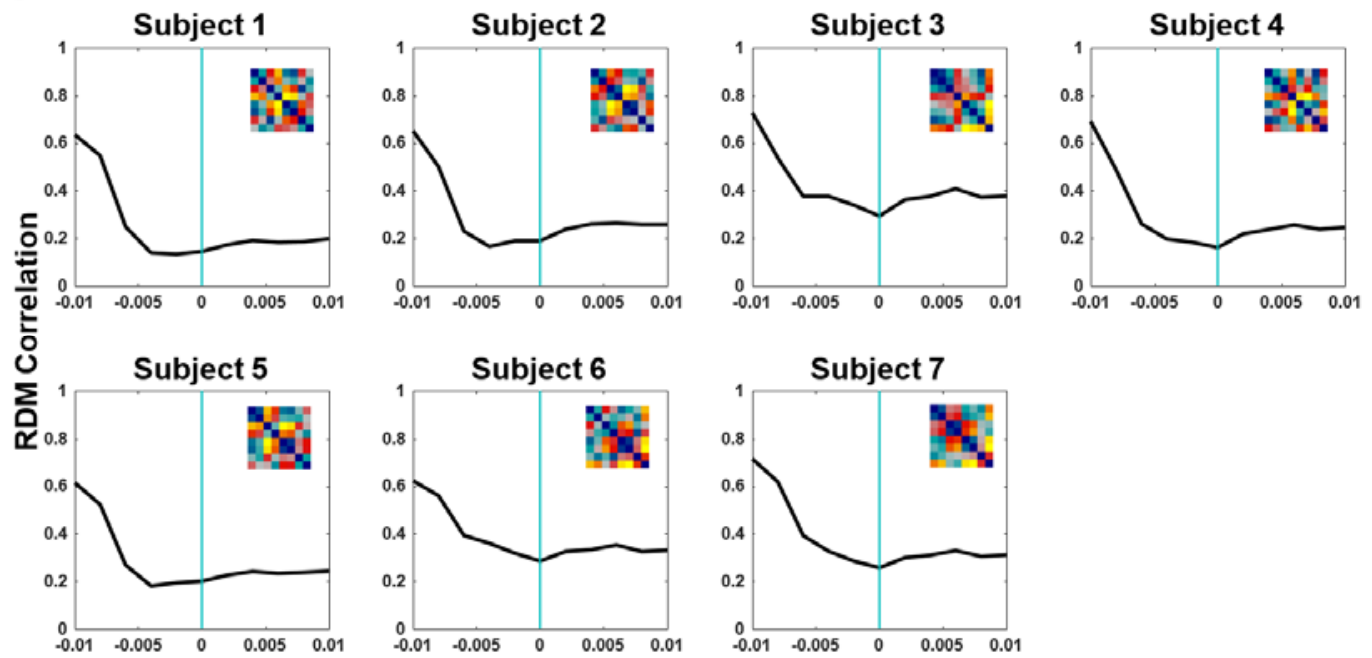

Offset value 


\section{Figure 4. Strict forward model provides better fit to the RT data in monkeys but}

not in humans. (A) "Offsets" are defined as the magnitude of reduced RT when the frames were in Clip 2. 11 hypothetical models with their reaction time patterns (top) and RDMs (bottom). We systemically varied the "offset" parameter while keeping a constant slope. These models progressively range from an absolute Global compression model (model 1, most left), to a Strict-forward model (model 6, middle), and beyond ( $7^{\text {th }}$ to $11^{\text {th }}$ models, right). The numerals below the RDMs denote the magnitude of the respective offsets. (B) Each monkey's data is tested against each of these 11 hypothetical models. The Spearman correlations increase as a function of offset magnitude between Clip 1 and Clip 2 until reaching an asymptote when the offset value is around zero, which corresponds to the Strict forward model (model 6 in Figure 4A; see also Figure 3). Individuals' RT RDMs are shown in insets. (C) Each human participant's data is also tested against each of these 11 hypothetical models. The Spearman correlations decrease as a function of offset magnitude between Clip 1 and Clip 2 until reaching an asymptote when the offset value is around zero. This confirms the hypothetical discrepancy between the two species (see also Figure 3B).

We have considered a further factor - slope of RT/chosen frame location - in our model, which might be relevant for the question at hand. To take negative slopes into consideration, we calculated RDM based on the relative differences of each pair of segments. Then, we transformed the vectors "offset" and "slope of RT/chosen frame location" into two arrays and displayed them as three-dimensional mesh/surface plots. In Figure 5, in addition to "offsets", we showed that the correlation between model and monkeys' data also increase as the slope of RT/chosen frame location increases. In contrast, the correlation values for human data seem to be driven by the "offsets" (cf. Figure 4C), and in terms of "slope" going in an opposite direction from the monkeys (cf. Figure 2A-B). This visualization converges with other current findings

342 that the slope of RT/chosen frame location does also matter to highlight the 
A
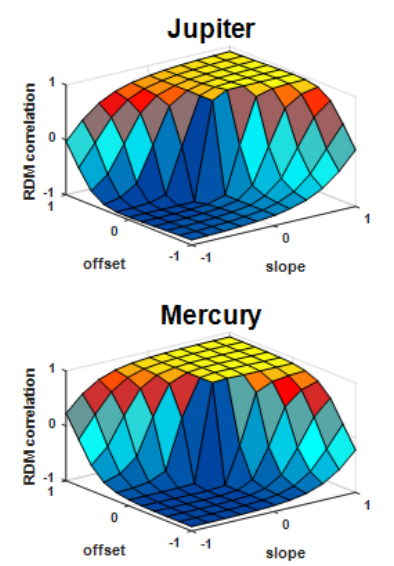
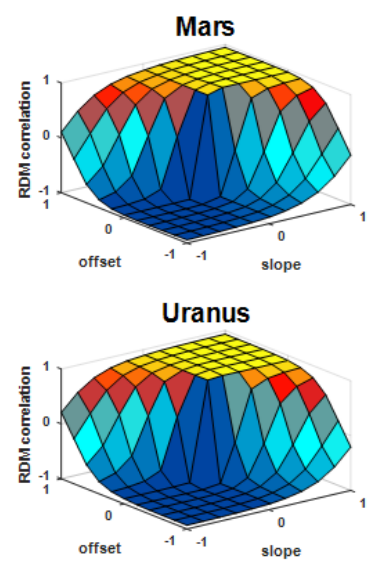
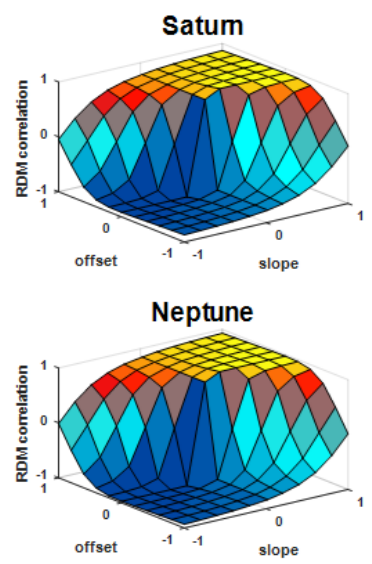

B

Subject 1

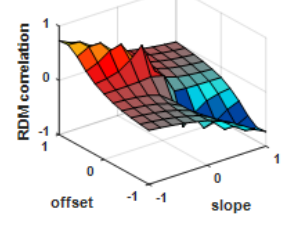

Subject5

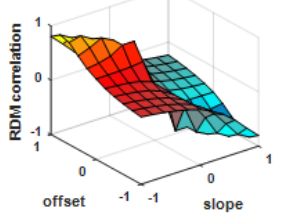

Subject 2

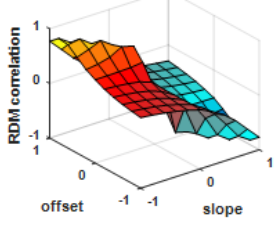

Subject 6

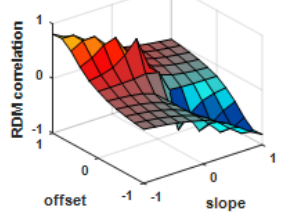

Subject 3

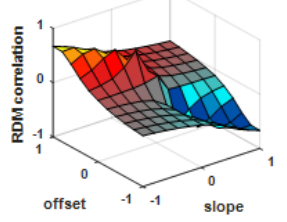

Subject 7

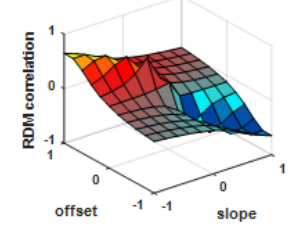

Subject 4

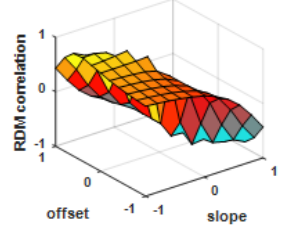

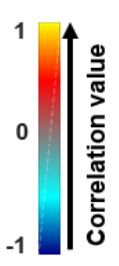

Figure 5. Visualization for RDM correlation as modulated by "offset" and "slope" in three-dimensional space (slope, offset, RDM correlation). (A) Monkeys data (B)

\section{Context changes (event boundary) increase rate of rise in decision information}

We have thus far focused on how the monkeys retrieve the order of frames when information was equated within contexts, but how contextual changes would aid temporal order judgement processes remains to be examined. It was evident that the monkeys retrieved the temporal order of frames with numerally different speeds for the three trial-types, across- vs. within-Clip1 vs. within-Clip2: $F(2,15)=2.32, P=$ 
360

361

362

363

364

365

366

367

368

369

370

371

372

373

374

375

376

377

378

379

380

381 within-context and across-context conditions and hypothesized that a context shift would change the rate of rise of information accumulation (shift model) without altering the decision threshold (swivel model) with the Linear Approach to Threshold with Ergodic Rate (LATER) model (Figure 6A). We compared across-context and within-context trials specifically and fitted the two types of LATER models separately on each monkey's data (Figure 6B), together with an unconstrained model, which supposes the reaction times of two conditions are independent from each other, and a null model, which assumes there is no effect of manipulation. Using the Bayesian information criterion (BIC) as an index of model comparison, the results consistently indicate that the shift model is better than the swivel model in all six monkeys (range of $\triangle \mathrm{BIC}=[14.57,300.07]$; Table S1). These results further indicate that contextual changes do not alter the judgement threshold for decisions (no evidence for a swivel pattern). Within a drift diffusion model framework, the results suggest that monkeys accumulate information for memory decisions at a faster rate when the frames were extracted from two different clips than for frames that were extracted from the same-context clip, signifying a boundary effect in memory in macaque monkeys which parallel closely those established findings in the humans (17). These results suggest that the monkeys adopt a forward search for targets among linearly ordered memory traces and they reach their memory decision threshold more quickly when probe frames are extracted from the two different contexts (across-context condition). 
A

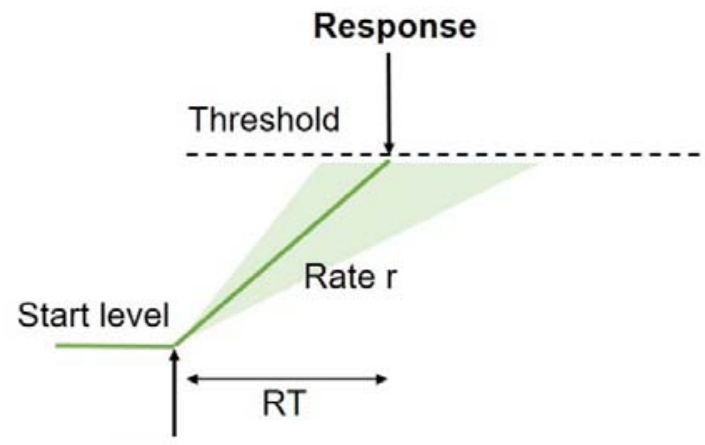

Stimulus

B

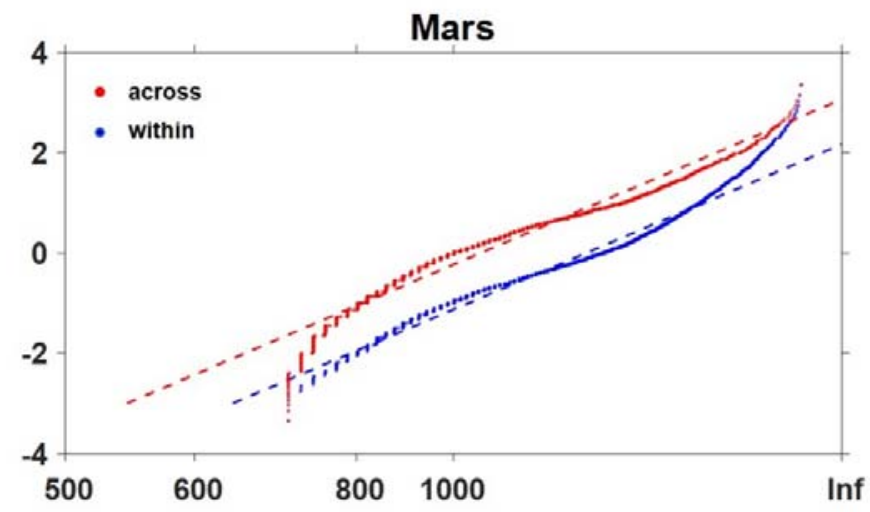

Mercury

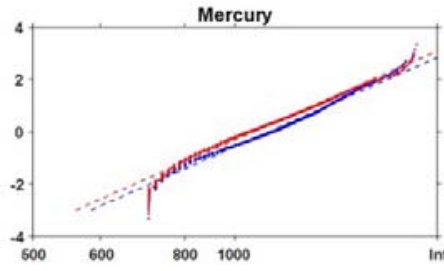

Uranus
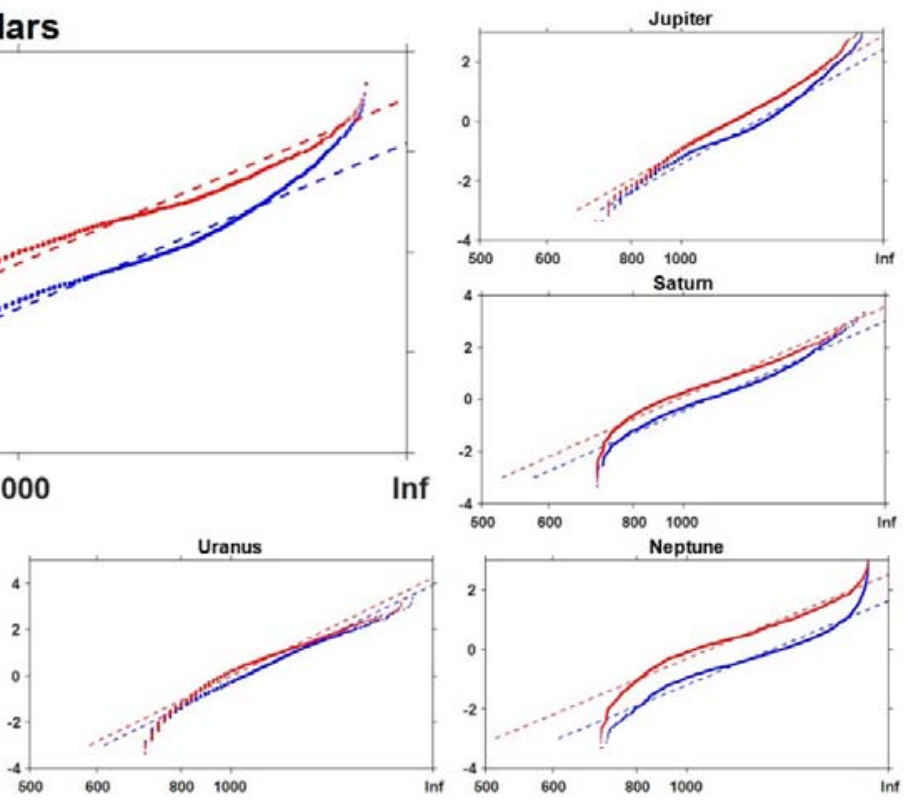

383

384

385

386

387

388

389

390

391

392

393

\section{Figure 6. LATER model fitting of RT in across-context and within-context}

condition. (A) The LATER model cartoon depicts that a decision signal triggered by a stimulus rises from its start level, at a rate of information accumulation $r$, to the threshold. Once it reaches the threshold, a decision is initiated. The rate of rise $r$ varies from trial to trial, obeying the Gaussian distribution (variation denoted as green shaded area). (B) Contextual change effect on the distribution of response latency for the monkeys; data from Monkey "Mars" was chosen for larger display. The red and blue dash lines show the best fits (maximum likelihood) of across-context trials and within-context trials respectively. 


\section{Confirmatory GLMs for the putative patterns}

In order to verify whether the effects are not attributed to the basic stimulus features such as perceptual differences inherent in the across-context condition. We then performed several generalized linear models to quantify the effect sizes of several principal variables. In within-context condition, given that the monkeys would replay their experience to judge the relative temporal order of probe frames ("replay hypothesis"), we used temporal characteristics of probe frames, as represented by chosen frame location (or temporal similarity, which is essentially an inverse of frame location) as the independent variables. In across-context condition, we included a perceptual similarity measure based on feature points extracted by the SURF algorithm (SURF similarity, Figure S2) into the generalized linear model to reflect the extent to which the monkeys could capitalize on using contextual boundaries for TOJ judgment. The within-context GLM shows that monkey's RT was indeed significantly faster when the probe frame was located earlier in the video, $P<0.001$ (or in equivalent terms, when two frames were temporally closer, $P=0.004)$, confirming our main finding that

410 the monkeys adopt a forward scanning strategy for information retrieval (Figure 7 left).

411 In contrast, the across-context GLM shows that there was not any significant effect of 412 the chosen frame location on RT. Rather, the monkeys retrieve their memories 413 significantly faster for probe frames that are contextually (or perceptually) distinct, $P=$ 4140.004 (Figure 7 right). These results are fully replicated when several more regressors 415 which can potentially affect RT are additionally entered into the design matrix (Figure 416 S3). 
417

418

419

420

421

422

423

424

425

426

427

428

429

430

431

432

433
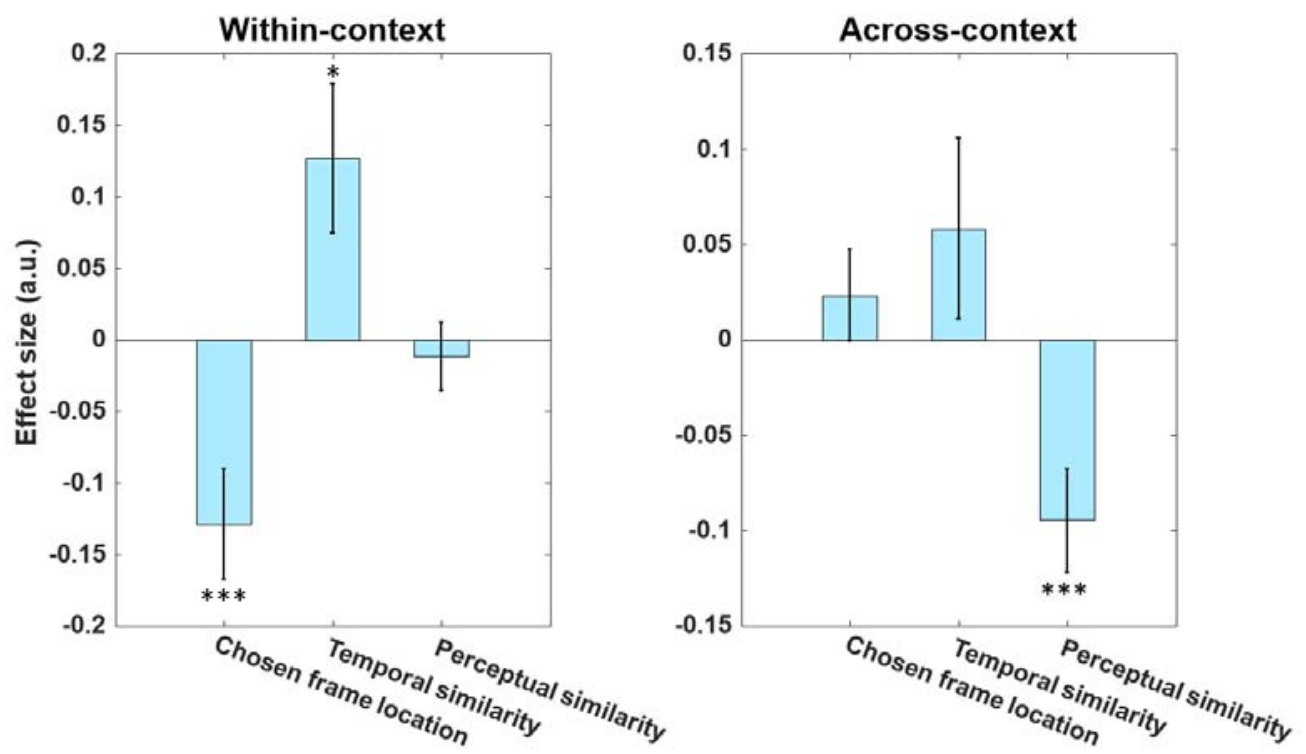

Figure 7. GLM results on the effects of model variables on reciprocal latency for

within-context and across-context condition. The chosen frame location and the temporal similarity are variables associated with the replay hypothesis, whereas the perceptual similarity is with the context hypothesis. In within-context condition, the chosen frame location and the temporal similarity both show significant effects on RT, $P<0.001$ and $P=0.004$ respectively, wheras the perceptual similarity does not, $P=$ 0.844. We found an opposite effect pattern in across-context condition. The perceptual similarity shows a significant effect on RT, $(P=0.004)$, while the chosen frame location $(P=0.796)$ and the temporal similarity $(P=0.446)$ do not. Effect size (in arbitray unit, a.u.) denote the beta values. Error bars indicate SEM . *** denotes $P<$ 0.001 , ** denotes $P<0.01$, * denotes $P<0.05$. See also Figure $\mathbf{S 3}$ for a GLM analysis including a full range of variables. 


\section{DISCUSSION}

In light of recent reports on neural correlates underlying how humans and rodents reply their past experiences $(11,12,14,18)$, here, we demonstrate parallel behavioral findings in macaque monkeys with dynamic cinematic material. Previous reports of macaques succeeding in temporal order judgement indicated their ability in remembering the order of events $(1,5,19)$ and even meta-cognitively monitor the quality of representations of temporal relations among item images (20). For example, Orlov et al. (8) suggested that monkeys can categorize stimuli by their ordinal number to aid recall of order, and Templer et al. showed that monkeys retrieve the temporal order information based on the order of events rather than elapsed time (1). One possible common mechanism underlying these performances is that monkeys use a forward search to identify targets in memory representation (2). Taking advantage of the latency data obtained during TOJ on naturalistic materials, we provide new behavioral evidence in support of the hypothesis proposed by Gower (2) that the monkeys can replay their memory in a serial forward manner. Our analysis further clarifies that this replay process is conducted in a time-compressed manner. Notwithstanding task differences, both humans and macaques execute retrieval with forward replay with a comparable compression factor (factors of $\sim 11$ in macaques vs. $\sim 13$ in humans, cf. (12), but see also (13)).

Despite the cross-species similarity, our revelation of a critical discrepancy between humans and macaques carries an important theoretical implication: humans can do both local and global compression whereas monkeys are not able to attain global compression. The implication is that mental time travel is not all-or-none. There could be multiple layers underpinning the concept of mental time travel, which entail the ability to relive the past $(21,22)$ and skipping over unimportant information (12). The latter aspect allows humans to recall our memories flexibly far into our past and suggest powerful computational efficiencies that may facilitate memory storage and recall. In contrast, we did not find any evidence in the macaques for their ability of making use of salient boundary cues to skip unimportant details. It thus remains 
unknown in the current experiment how far back in time monkeys can replay their memories. It has been recently shown that humans can spontaneously replay experience based on learned structures, with fast structural generalization to new experiences by representing structural information in a format that is independent from its sensory consequences (11). The lack of global compression in the monkeys of their video experience implies that the monkeys might not be able to use factorized representations to allow components to be recombined in more ways than were experienced (23).

A further caveat is that our monkeys perform their replay-like recall of the videos as an effortful operation to solve a TOJ task whereas most of the extant studies on replay focus on spontaneous replay patterns at rest (11), during sleep $(24,25)$ or during task-free state $(26,27)$. While we have argued for the presence of replay-like patterns in the monkey during TOJ, we are aware that replay is a neural phenomenon supported by activity of individual neurons and implicates offline reactivation of sequences of hippocampal place cells that reflect past and future trajectories (28-30). However, by establishing that neither the number of intervening frames nor passage of time per se determines the RT pattern (probably a mixed effect resultant from a combination of both), we ruled out order- or positional- memory as the underlying mechanism supporting TOJ in this task. Our results combined thus provide a novel connection between various kinds of replay(-like) behaviors linking between rodents and humans and provide a primate model for neuronal investigation.

We observed one further interesting feature here that the monkeys are able to detect contextual changes to facilitate TOJ. We show that the expedited TOJ in across-context condition was facilitated by contextual details, which in turn results in an increased rate of rise of signal towards memory decision in a drift-diffusion process. Humans studies show that contextual changes lead to segmentation of ongoing information $(31,32)$. Our results provide evidence consistent with event segmentation in the macaque monkeys and imply that macaque monkeys might be capable of parsing the footage by contextual information, akin to what has been shown in humans (33-36) and rodents (37). 
493 Memory replay is an elaborate mental process and our demonstration of a 494 time-compressed, forward replay-like pattern in the macaque monkeys provides 495 insights into mapping the mechanisms and evolution of episodic memory in our 496 lineage. There are however limitations in their ability in compressing the experienced 497 past globally, which somehow indicates a form of primordial mnemonic rigidity. This 498 cognitive discrepancy in our lineage should be further elucidated via 499 electrophysiological or neuroimaging methods probing into the MTL $(18,26,38,39)$ 500 and the neocortices (16). 
501

502

503

504

505

506

507

508

509

510

511

512

513

514

515

516

517

518

519

520

521

522

523

524

525

526

527

528

529

\section{METHODS}

\section{Task performance}

The six monkeys performed the task with a significantly above chance level with an overall accuracy at $67.9 \pm 1.5 \%$ (mean \pm SD) and with an above chance accuracy for within-context trials, $t 5=14.35, p<0.001$. The human participants performed the task on average at $92.7 \pm 1.2 \%$.

\section{Subjects}

Macaque monkeys. Six male rhesus macaques (Macaca mulatta) $(5.49 \pm 0.5 \mathrm{~kg})$ with a mean age of 3.5 years at the start of testing participated in this study. They were initially housed in a group of 6 in a specially built spacious enclosure (max capacity = 12-16 adults) with enrichment elements such as a swing and climbing structures until the present study began. The monkeys were then housed in pairs during experimentation period according to their social hierarchy and temperament. They are fed twice a day with portions of 180-g monkey chow and pieces of fruits (8:30am/4:00pm). Water is available ad libitum except on experimental days. They are routinely offered treats such as peanuts, raisins and various kinds of seeds in their home cage for forage purpose. The monkeys were procured from a nationally accredited colony located in Beijing outskirts, where the monkeys were bred and reared. The animals are thus ecologically naive to the natural wilderness and should not have had any previous encounter with other creatures except humans and their companion. The room wherein they are housed is operated with an automated 12:12 (7am/7pm) light-dark cycle and kept within temperate around $18-23^{\circ} \mathrm{C}$ and humidity of $60-80 \%$.

Human subjects. Seven participants (mean age $=19.57 \pm 1.13,6$ female) took part in the experiment. The participants were recruited from the undergraduate population in East China Normal University. The participants provided informed consent and were compensated $400 \mathrm{RMB}$ for their time. We used the 6 unique video-trials sets and TOJ frames (one unique set per monkey) correspondingly for the human subjects (subject 7 re-used set 1). 
The experimental protocol was approved by the Institutional Animal Care and Use Committee (permission code: M020150902 \& M020150902-2018) and the University Committee on Human Research Protection (permission code: HR 023-2017) at East China Normal University. All experimental protocols and animal welfare adhered with the "NIH Guidelines for the Care and Use of Laboratory Animals".

\section{Apparatus and testing cubicle}

The testing was conducted in an automated test apparatus controlled by two Windows computers (OptiPlex 3020, Dell). The subject sat, head-unrestrained, in a wheeled, specially-made Plexiglas monkey chair $(29.4 \mathrm{~cm} \times 30.8 \mathrm{~cm} \times 55 \mathrm{~cm})$ fixed in position in front of a 17-inch infrared touch-sensitive screen (An-210W02CM, Shenzhen Anmite Technology Co., Ltd., China) with a refresh rate at $60 \mathrm{~Hz}$. The distance between the subjects' head and the screen was kept at $\sim 20 \mathrm{~cm}$. The touch-sensitive screen was mounted firmly on a custom-made metal frame $(18.5 \mathrm{~cm} \times$ $53.2 \mathrm{~cm})$ on a large platform $(100 \mathrm{~cm} \times 150 \mathrm{~cm} \times 76 \mathrm{~cm})$. Water reward delivery was controlled by an automated water-delivery rewarding system (5-RLD-D1, Crist Instrument Co., Inc., U.S.) and each delivery was accompanied by an audible click. An infrared camera and video recording system (EZVIZ-C2C, Hangzhou Ezviz Network Co., Ltd., China) allowed the subject to be monitored while it was engaged in the task. The entire apparatus was housed in a sound-proof experimental cubicle that was dark apart from the background illumination from the touch screen.

\section{Source of video materials and preparation}

A collection of documentary films on wild creature was gathered from YouTube. The films were Monkey Kingdom (Disney), Monkey Planet (Episode 1 - 3; BBC), Planet Earth (Episode 1 - 11; BBC), Life (Episode 1 - 10; BBC), and Snow Monkey (PBS Nature). In total 28 hours of footage was gathered. We applied Video Studio X8 (Core Corporation) to parse the footage by camera-cuts into smaller segments. Experimenters then applied the following criteria to manually edit out $\sim 2500$ unique clips: 1) the clip must contain a continuous flow of depiction of events (i.e., no scene 
560 transition); 2) at least one living creature must be included; 3a) at least one of the

561 animals contained must be in obvious motion; $3 b$ ) the trajectories of these motion must

562 be unidirectional (i.e., no back and forth motion of the same subject); 4) clips with

563 snakes were discarded. From this library, we then selected 2000 clips (all of $4 \mathrm{~s}-6 \mathrm{~s}$ )

564 for the final test and a small number of additional clips were also prepared for the

565 training stages. For each individual monkey, the videos assigned to be in an

566 experimental condition would not be used/shown in another condition so that a

567 particular monkey will only view that video repeatedly under the same condition

568 across exposure.

569

570 Task and experimental procedure

571 We combined naturalistic material with a temporal order judgement paradigm that

572 is widely used in episodic memory research $(1,40,41)$. In each trial, the monkey

573 initiated a trial by pressing a colored rectangle in the center of the screen $(0.15 \mathrm{ml}$

574 water). An 8-12 s video (consisting of two 4-6 s clips) was then presented $(0.15 \mathrm{ml}$

575 water), and following a 2-s retention delay, two frames extracted from the video were

576 displayed bilaterally on the screen for TOJ. The monkeys were trained to choose the

577 frame that was shown earlier in the video (see Movie S1). A touch to the target frame

578 resulted in $1.5 \mathrm{ml}$ water as reward, removed the foil frame, and the target frame would

579 remain alone for $5 \mathrm{~s}$ as positive feedback. A touch to the foil frame removed both

580 frames from the screen and blanked the screen for $20 \mathrm{~s}$ without water delivery. Since

581 the monkeys could self-start the trials, we did not set an explicit inter-trial interval.

582 Correction trial procedures were not used in the main test.

583 We collected 50 daily sessions of data. Each session contained 100 trials, giving us

584 a 5000 trials per monkey. The 5000 trials contained a break-down of four factors:

585 Boundary (Within vs. Across), Play Order (Normal vs. Reverse), Temporal distance

586 (TDs, 25 levels), and Exposure (R1 - R5), giving out a $2 * 2 * 25 * 5$ within-subject

587 design. The two frames for TOJ could be extracted from same clip (Within) or distinct

588 clips (Across). The 25 levels of TDs ranged between a minimum of $1000 \mathrm{~ms}$

589 (equivalent of 25 frames) and a maximum of $3880 \mathrm{~ms}$ (equivalent of 97 frames). Each 
590

591

592

593

594

595

596

597

598

599

600

601

602

603

604

605

606

607

608

609

610

611

612

613

614

615

616

617

618

619

TD level increased progressively with 3 frames each step. The 10 lists contained 5 lists

of primate animals and 5 lists of non-primate animals (Category: Primate/Non-Primate).

The 6 monkeys were counter-balanced in their order of receiving the two kinds of material: three monkeys (Jupiter, Mars, and Saturn) were tested first on Non-Primate lists, whereas the other three (Mercury, Neptune, and Uranus) were tested first on Primate lists. The whole experiment lasted for 68 days. There were three blocks of reset (3 days, 9 days and 6 days) in-between testing days. The task was programmed in in PsychoPy2 implemented in Python.

\section{Data analysis, temporal and perceptual similarity, and model comparison}

\section{Representational similarity analysis (RSA)}

To create RDMs for the representational pattern of response time, we evenly divided each video into eight segments based on chosen frame location and averaged the response times within each segment for each monkey individually. For each matrix, we then computed the Euclidean distance of average response time for each pair of the segments. In order to show the relative distance among all pairs of segments intuitionally, we further transformed the matrix by replacing each element with the rank number in the distribution of all the elements, and linearly scaled into $[0,1]$. To compare similarity between response times patterns and the candidate models, we computed Spearman correlation between the model and group averaged RDMs with 100 randomized iterations. The statistical threshold was set at $p<0.05$ (FDR corrected).

\section{LATER (linear approach to threshold with ergodic rate) modelling}

The observations that the brain needs longer time than it requires for nerves to transport information and that trial-by-trial RTs vary considerably have stimulated researchers to make use of distribution of reaction times for examining mental processes (37). Latency, as an indicator of decision processes, provides a source of insight into the underlying decision mechanisms (34-36). The Linear Approach to Threshold with Ergodic Rate (LATER) model is a widely used model that taps into 
these processes (42). The LATER model stipulates that the winner signals that reach threshold faster would trigger the decision, resulting in shorter latency. Accordingly, changing the rate of rise would cause the line to "shift" along the abscissa without changing its slope. In contrast, lines plotted by latency distribution would "swivel" around an intercept when the threshold changes (43) (Figure 4A). To explore the underlying mechanisms of different experiment conditions, we plotted the reciprocal of reaction time as a function of their z-scores, on account for making the distributions follow Gaussian distribution (44). Hence, we fitted the main component for each condition. The main component is a ramp to the threshold with rate of rise $r$. The distance between start level $\mathrm{S}_{0}$ and threshold $\mathrm{S}_{\mathrm{T}}$ is defined as $\theta$, and rate of rise $r$ follows a Gaussian distribution of mean, $\mu$, and standard deviation, $\sigma l$.

For model comparison, we fitted four different models to the data. The "null" model fits reaction times of both conditions with the same parameters, implying no effect of manipulation. The "Unconstrained" model set all the parameters to be free, which supposes the reaction times of two conditions are independent from each other. The "Shift" model only allows the slope of main component $\mu$ to change according different conditions, on the assumption that the manipulation will change the rate of rise. The "Swivel" model only allows $\theta$ to change according different conditions, on the assumption that the subject set different thresholds for different conditions.

\section{Generalized linear models (GLM)}

We ran generalized linear models to compare the effect sizes of independent variables to the dependent variable. The mean $(\mu)$ of the outcome distribution Y depends on the independent variables $\mathrm{X}$, through the following formula:

$$
\mathrm{E}(\mathrm{Y})=\mu=g^{-1}(X \beta)
$$

where $\mathrm{Y}$ is a distribution of outcomes, $\beta$ is unknown parameters to be estimated, $g$ is a link function (Gaussian function). The dependent variable (Y) is reciprocal latency, and independent variables are as follows: a binary regressor indicating whether the video includes primate content or not, a binary regressor indicating that a video is played forward or backward, the five repetitions of the video-trials, physical 
650 location of the selected probe (left or right), time elapsed within session, chosen frame

651 location, temporal similarity, perceptual similarity (SURF), and temporal distance. To

652 reduce the effect of behavioral noise of monkeys on the analysis, we included only

653 correct trials in these GLM analyses and excluded trials with RT longer than $10 \mathrm{~s}$ or

654 shorter than $0.7 \mathrm{~s}$.

655

Model comparison

657 To obtain the best fit among these models, we used Bayesian Information 658 Criterion (BIC) as a criterion for model selection among these four models. The 659 formula for $\mathrm{BIC}$ is: $\mathrm{BIC}=-2(\log \mathrm{L})+$ numParam* $\log ($ numObs $)$, where $\mathrm{L}$ is the 660 maximum likelihood for the model, numParam and numObs represent the number of 661 free parameters and the number of samples respectively. We computed $\triangle \mathrm{BIC}$ as the 662 strength of the evidence, which indicates to what extent the selected model is superior 663 to other models. Different ranges of $\triangle \mathrm{BIC}$ show different level of evidence: The value 664 of $\triangle \mathrm{BIC}$ larger than 2 shows a positive evidence, the value of $\triangle \mathrm{BIC}$ larger than 6 665 indicates a strong evidence (56).

666

\section{Temporal similarity}

668 For each trial, we calculated temporal similarity (TS) as an index of the 669 discriminability of probe frames. Temporal similarity between two probe frames 670 extracted from the video is calculated by the ratio of two frames' temporal separation 671 between their occurrence in the video and the time of testing. Temporal similarity of 672 any two memory traces can be calculated as: TS = delay 2 / delay 1 , where delay $2<$ 673 delay1 (45).

Perceptual similarity

RGB-histogram is computed as the Sum-of Square-Difference (SSD) error 677 between image pairs for the three color channels (RGB). For each color channel the 678 intensity values range from 0 to 255 (i.e., 256 bins), we first computed the total number 679 of pixels at each intensity value and then computed the SSD for all 256 bins for each 
680 image pair. The smaller the value of the SSD, the more similar 658 the two images

681 (image pair) was. In the case of the HOG similarity, we constructed a histogram of

682 directions of gradient over fixed sized grid across the entire image. A vector is

683 generated from each grid cell and correlated with HOG features from another image.

684 Besides, Speeded Up Robust Features (SURF) (46) uses Box Filter which is

685 calculated in parallel using integral images (47) to approximate

686 Laplacian-of-Gaussian (LoG). Wavelet responses in both horizontal and vertical

687 directions are used to assign orientation in SURF. The first step in SURF consists of

688 fixing a reproducible orientation based on information from a circular region around

689 the interest point. A descriptor vector is generated around the interest point using

690 integral image, which is compared to descriptor vectors extracted from a compared

691 image to find a match. The Euclidean distance is used to measure the similarity between

692 two descriptor vectors from two images.

693

694

Data preprocessing

695 Trials with RT longer than $10 \mathrm{~s}(1.45 \%)$ or faster than $0.7 \mathrm{~s}(2.47 \%)$ were 696 excluded from the analyses. Both correct and incorrect trials were entered for all 697 analyses, except those reported in the GLMs. For Neptune, data of one day of primate 698 list 5 (exposure 4) and two days of primate lists 4 and 5 (exposure 5) were lost due to 699 machine breakdown and thus only 4,700 trials are included for Neptune. 
702 Funding: This research received funding from National Key Fundamental Research

703 Program of China (973 Program) Grant 2013CB329501 (Y-d Z.), Ministry of

704 Education of PRC Humanities and Social Sciences Research Grant 16YJC190006

705 (S.C.K.). Author contributions: Conceptualization, S.Z., L.W. and S.C.K.;

706 Methodology, S.Z., L.W., J.S., S.W.L., K.A., and S.C.K.; Investigation, S.Z., L.W.,

707 and Y.C.; Data interpretation: S.Z., L.W., J.S., S.W.L., Y-d.Z., and S.C.K.; Writing -

708 Original Draft, S.Z. and S.C.K.; Writing - Review \& Editing, S.Z. and S.C.K.; Funding

709 Acquisition, Y-d.Z., and S.C.K.; Supervision, S.C.K. Competing interests: Authors

710 declare no competing interests. Data and materials availability: All data is available

711 at Dryad (doi: 10.5061/dryad.3r2280gcc) and codes are available upon request. 


\section{REFERENCES}

1. V. L. Templer, R. R. Hampton, Cognitive mechanisms of memory for order in rhesus monkeys (Macaca mulatta). Hippocampus. 23, 193-201 (2013).

2. E. C. Gower, Short-term memory for the temporal order of events in monkeys. Behav. Brain Res. 52, 99-103 (1992).

3. D. P. Charles, D. Gaffan, M. J. Buckley, Impaired Recency Judgments and Intact Novelty Judgments after Fornix Transection in Monkeys. J. Neurosci. 24, 2037-2044 (2004).

4. N. Morimura, T. Matsuzawa, Memory of movies by chimpanzees (Pan troglodytes). J. Comp. Psychol. 115, 152-158 (2001).

5. G. Martin-Ordas, D. Haun, F. Colmenares, J. Call, Keeping track of time: Evidence for episodic-like memory in great apes. Anim. Cogn. 13, 331-340 (2010).

6. H. S. Terrace, L. K. Son, E. M. Brannon, Serial expertise of rhesus macaques. Psychol. Sci. 14, 66-73 (2003).

7. S. Chen, K. B. Swartz, H. S. Terrace, Knowledge of the Ordinal Position of List Items in Rhesus Monkeys. Psychol. Sci. 8, 80-86 (1997).

8. T. Orlov, V. Yakovlev, S. Hochstein, E. Zohary, Macaque monkeys categorize images by their ordinal number. Nature. 404, 77-80 (2000).

9. V. L. Templer, R. P. Gazes, R. R. Hampton, Co-operation of long-term and working memory representations in simultaneous chaining by rhesus monkeys ( Macaca mulatta ). Q. J. Exp. Psychol. (2019), doi:10.1177/1747021819838432.

10. N. M. Long, M. J. Kahana, Hippocampal contributions to serial-order memory. Hippocampus. 29, 252-259 (2019).

11. Y. Liu, R. J. Dolan, Z. Kurth-Nelson, T. E. J. Behrens, Human Replay Spontaneously Reorganizes Experience. Cell, 1-13 (2019).

12. S. Michelmann, B. P. Staresina, H. Bowman, S. Hanslmayr, Speed of time-compressed forward replay flexibly changes in human episodic memory. Nat. Hum. Behav. 3, 143-154 (2019). 
13. G. E. Wimmer, Y. Liu, N. Vehar, T. E. Behrens, R. J. Dolan, Episodic memory retrieval is supported by rapid replay of episode content. bioRxiv, 758185 (2019).

14. D. Panoz-Brown et al., Replay of Episodic Memories in the Rat. Curr. Biol. 28, 1628-1634.e7 (2018).

15. K. Bonasia, J. Blommesteyn, M. Moscovitch, Memory and navigation: Compression of space varies with route length and turns. Hippocampus. 26, 9-12 (2016).

16. Y. Naya, H. Chen, C. Yang, W. A. Suzuki, Contributions of primate prefrontal cortex and medial temporal lobe to temporal-order memory. Proc. Natl. Acad. Sci. 114, 13555-13560 (2017).

17. J. M. Zacks, B. Tversky, Event Structure in Perception and Conception. Psychol. Bull. 127, 3-21 (2001).

18. T. J. Davidson, F. Kloosterman, M. A. Wilson, Hippocampal Replay of Extended Experience. Neuron. 63, 497-507 (2009).

19. Y. Ninokura, H. Mushiake, J. Tanji, Representation of the Temporal Order of Visual Objects in the Primate Lateral Prefrontal Cortex. J. Neurophysiol. 89, 2868-2873 (2003).

20. V. L. Templer, E. K. Brown, R. R. Hampton, Rhesus monkeys metacognitively monitor memories of the order of events. Sci. Rep. 8, 11541 (2018).

21. T. Suddendorf, D. R. Addis, M. C. Corballis, Mental time travel and the shaping of the human mind. Philos. Trans. R. Soc. B Biol. Sci. 364, 1317-1324 (2009).

22. E. Tulving, Memory and consciousness. Can. Psychol. Can. 26, 1-12 (1985).

23. T. E. J. Behrens et al., What Is a Cognitive Map? Organizing Knowledge for Flexible Behavior. Neuron. 100, 490-509 (2018).

24. K. Louie, M. A. Wilson, Temporally structured replay of awake hippocampal ensemble activity during rapid eye movement sleep. Neuron. 29, 145-156 (2001). 
25. A. K. Lee, M. A. Wilson, Memory of Sequential Experience in the Hippocampus during Slow Wave Sleep. Neuron. 36, 1183-1194 (2002).

26. D. J. Foster, M. A. Wilson, Reverse replay of behavioural sequences in hippocampal place cells during the awake state. Nature. 440, 680-683 (2006).

27. M. P. Karlsson, L. M. Frank, Awake replay of remote experiences in the hippocampus. Nat. Neurosci. 12, 913-918 (2009).

28. B. E. Pfeiffer, D. J. Foster, Hippocampal place-cell sequences depict future paths to remembered goals. Nature. 497, 74-79 (2013).

29. S. P. Jadhav, C. Kemere, P. W. German, L. M. Frank, Awake Hippocampal Sharp-Wave Ripples Support Spatial Memory. Science (80-. ). 336, 1454 (2012).

30. H. F. Ólafsdóttir, D. Bush, C. Barry, The Role of Hippocampal Replay in Memory and Planning. Curr. Biol. 28, R37-R50 (2018).

31. J. P. Magliano, J. Miller, R. A. Zwaan, Indexing Space and Time in Film Understanding. Appl. Cogn. Psychol. 15, 533-545 (2001).

32. B. M. Hard, B. Tversky, D. S. Lang, Making sense of abstract events: Building event schemas. Mem. Cogn. 34, 1221-1235 (2006).

33. S. DuBrow, L. Davachi, The influence of context boundaries on memory for the sequential order of events. J. Exp. Psychol. Gen. 142, 1277-1286 (2013).

34. I. Sols, S. DuBrow, L. Davachi, L. Fuentemilla, Event Boundaries Trigger Rapid Memory Reinstatement of the Prior Events to Promote Their Representation in Long-Term Memory. Curr. Biol. 27, 3499-3504.e4 (2017).

35. Y. Ezzyat, L. Davachi, What constitutes an episode in episodic memory? Psychol. Sci. 22, 243-252 (2011).

36. S. C. Kwok, E. Macaluso, Exogenous features versus prior experiences modulate different subregions of the right IPL during episodic memory retrieval. Sci. Rep. 5, 11248 (2015).

37. D. Panoz-Brown et al., Rats Remember Items in Context Using Episodic Memory. Curr. Biol. 26, 2821-2826 (2016). 
801 38. M. P. Karlsson, L. M. Frank, Awake replay of remote experiences in the

802

803

804

805

806

807

808

809

810

811

812

813

814

815

816

817

818

819

820

821

822

823

824

825

826

827

828

829

830 hippocampus. Nat. Neurosci. 12, 913-918 (2009).

39. K. Diba, G. Buzsáki, Forward and reverse hippocampal place-cell sequences during ripples. Nat. Neurosci. 10, 1241-1242 (2007).

40. J. R. Manns, M. W. Howard, H. Eichenbaum, Gradual Changes in Hippocampal Activity Support Remembering the Order of Events. Neuron. 56, 530-540 (2007).

41. S. C. Kwok, E. Macaluso, Scale invariance of temporal order discrimination using complex, naturalistic events. Cognition. 140, 111-121 (2015).

42. I. Noorani, R. H. S. Carpenter, The LATER model of reaction time and decision. Neurosci. Biobehav. Rev. 64, 229-251 (2016).

43. B. A. J. Reddi, R. H. S. Carpenter, The influence of urgency on decision time. Nat. Neurosci. 3, 827-830 (2000).

44. I. Noorani, R. H. S. Carpenter, Full reaction time distributions reveal the complexity of neural decision-making. Eur. J. Neurosci. 33, 1948-1951 (2011).

45. G. D. A. Brown, I. Neath, N. Chater, A Temporal Ratio Model of Memory. Psychol. Rev. 114, 539-576 (2007).

46. H. Bay, A. Ess, T. Tuytelaars, L. Van Gool, Speeded-Up Robust Features (SURF). Comput. Vis. Image Underst. 110, 346-359 (2008).

47. P. Viola, M. J. Jones, Robust Real-Time Face Detection. Int. J. Comput. Vis. 57, 137-154 (2004). 


\section{Supplemental Information}

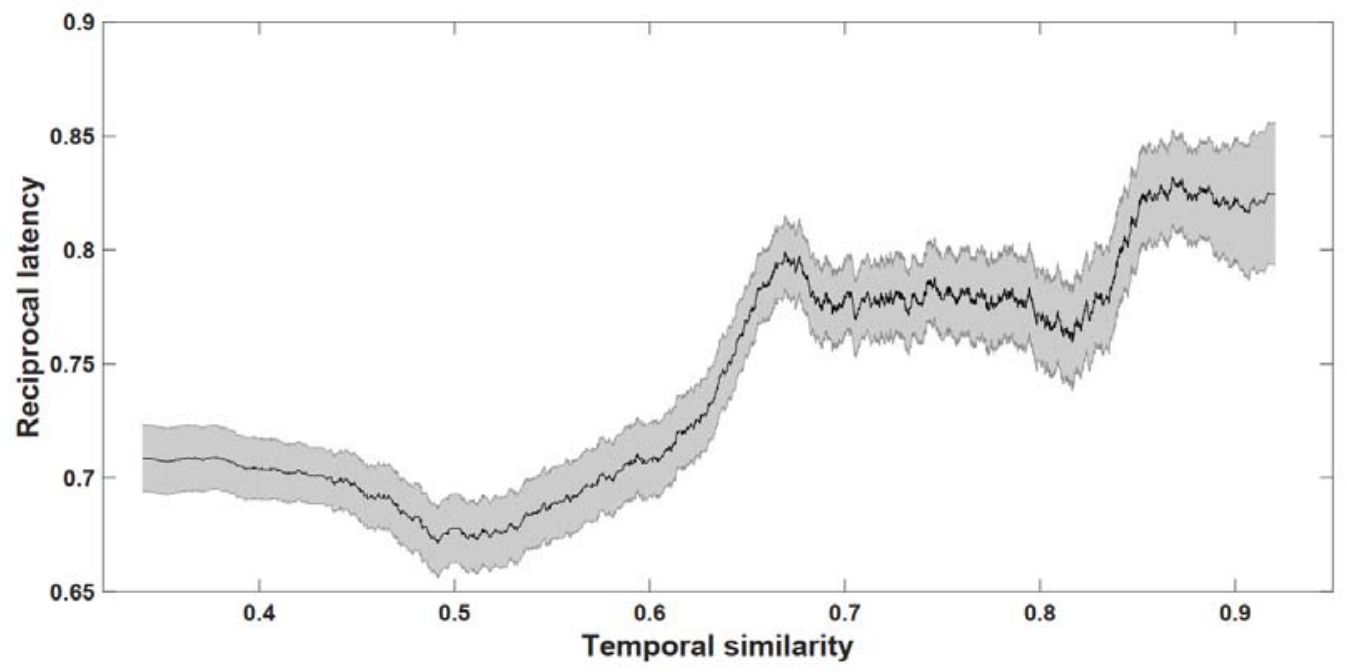

832
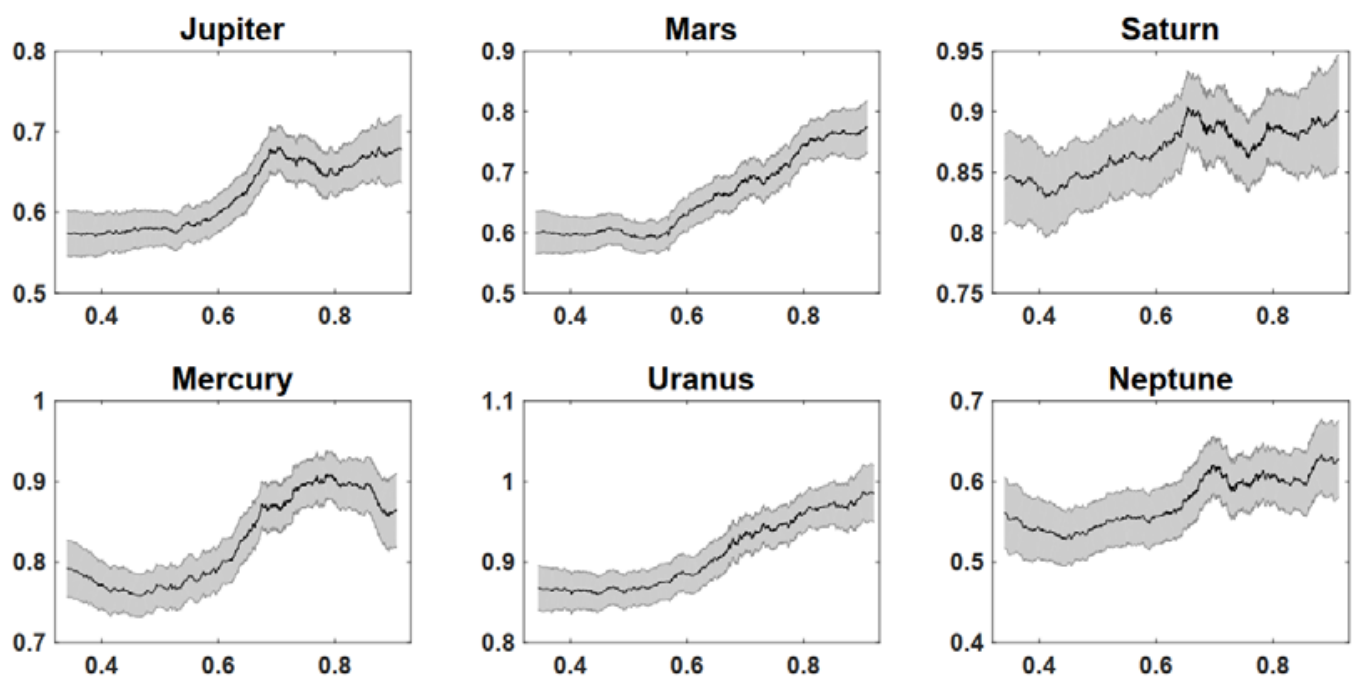

833

834

Figure S1. Relationship between temporal similarity and reciprocal latency for

836

within-context trials. Reciprocal latency as a function of temporal similarity for the average of all monkeys (upper panel) and individual monkeys (bottom panel). Temporal similarity between two frames is mathematically the inverse of their respective frame locations in the video, see Methods. Related to Figure 2A. 

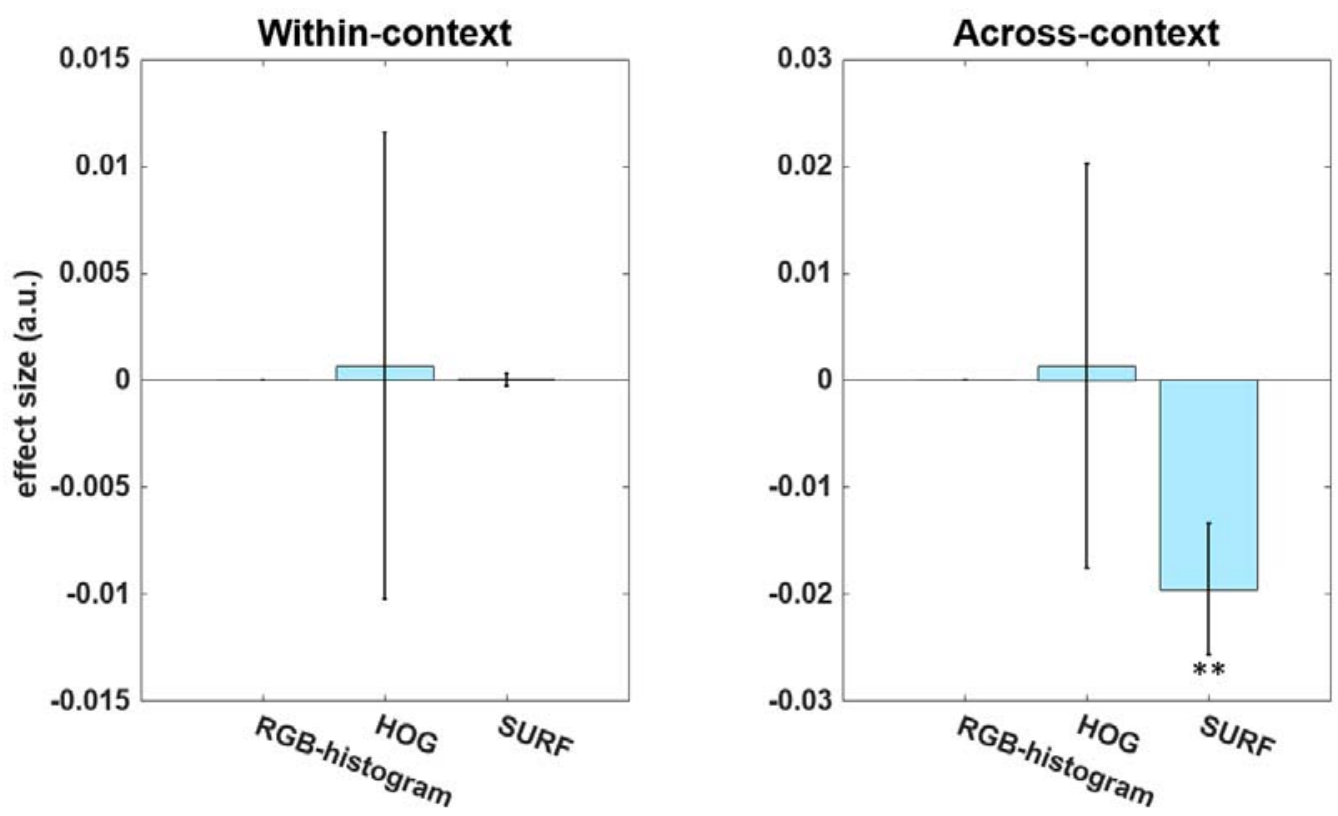

842

843

Figure S2. GLM results on the effects of image similarity measures on reciprocal

844 latency for within-context and across-context condition. Difference of distribution

845 in RGB- histogram, HOG similarity and SURF similarity were tested. The SURF

846 similarity measure was significantly correlated with RT in the across-context condition $(P=0.0015)$. Related to Figure 7. 
849

850

851

852

853

854

855

856

857

858

859

860

861

862

863

864

865

866

867

868
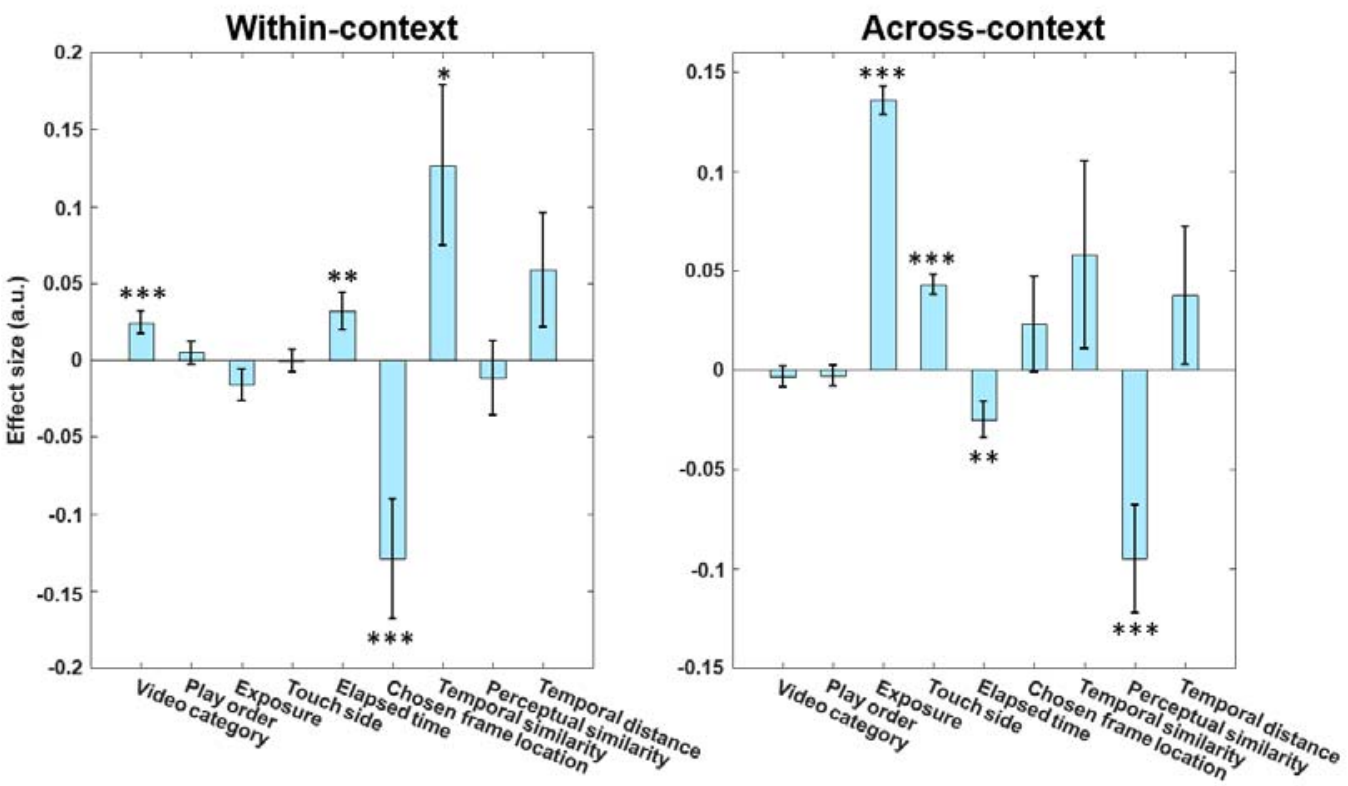

Figure S3. Full GLM analysis including a number of variables that might affect

reciprocal latency. The nine regressors include a binary regressor indicating whether the video category is primate or non-primate (video category), a binary regressor indicating that a video is played forward or backward (play order), the repeated exposure of the trial $(1-5)$ (exposure), the physical location of the selected probe on screen (left or right) (touch side), time elapsed within session (elapsed time; to rule out fatigue or attentional confounds), chosen frame location, temporal similarity, SURF similarity as a perceptual similarity measure (perceptual similarity) and temporal distance between two probe frames. The results confirm that chosen frame location is the most significant regressor in within-context trials while perceptual similarity is the most significant regressor in across-context trials. *** denotes $P<$ 0.001 , ** denotes $P<0.01$, * denotes $P<0.05$. Related to Figure 7 . 
870 Table S1. LATER model fitting results of six monkeys. For ease of comparison, we 871 computed the respective $\triangle \mathrm{BIC}$ to index the strength of evidence for each model. Note 872 that the model with the lowest BIC is the winning model. In all 6 monkeys, the shift 873 model was superior to the other three models. Related to Figure 6.

874

\begin{tabular}{|c|c|c|c|c|c|c|c|c|c|}
\hline \multirow{2}{*}{\multicolumn{2}{|c|}{ Condition }} & \multicolumn{4}{|c|}{ BIC } & \multicolumn{4}{|c|}{ delta_BIC } \\
\hline & & null & two fits & swivel & shift & null & two fits & swivel & shift \\
\hline \multirow{6}{*}{ Across VS Within } & Jupiter & -67157.39 & -67603.99 & -67543.25 & -67615.23 & 457.83 & 11.24 & 71.98 & 0 \\
\hline & Mars & -63948.06 & -65937.89 & -65769.84 & -65948.72 & 2000.65 & 10.82 & 178.88 & 0 \\
\hline & Satum & -61307.14 & -61929.72 & -61853.77 & -61938.70 & 631.56 & 8.98 & 84.93 & 0 \\
\hline & Mercury & -63904.96 & -64047.31 & -64042.94 & -64057.52 & 152.56 & 10.20 & 14.57 & 0 \\
\hline & Uranus & -67652.66 & -67850.84 & -67841.34 & -67864.67 & 212.01 & 13.83 & 23.34 & 0 \\
\hline & Neptune & -56695.53 & -58327.76 & -58037.15 & -58337.23 & 1641.70 & 9.47 & 300.07 & 0 \\
\hline
\end{tabular}

875

876 
877 Table S2. For monkeys: One sample $t$-tests results of the slopes of RT as a function of

878 temporal similarity. The three panels correspond to analyses performed using all trials

879 (top), only correct trials (middle), and only incorrect trials (bottom). Same slope

880 patterns were observed irrespective of correctness, in consistency with the analyses on

881 slopes of RT as a function of chosen frame location for each monkey. Related to

882 Table 1

883

884

\begin{tabular}{|c|c|c|c|c|c|c|c|}
\hline \multirow{2}{*}{ Monkeys } & \multirow{2}{*}{ Mean (SEM) } & \multirow{2}{*}{ t-statistics } & \multirow{2}{*}{ d.f. } & \multirow{2}{*}{$p$-value } & \multirow{2}{*}{ Cohen's d } & \multicolumn{2}{|c|}{$95 \%$ confidence interva } \\
\hline & & & & & & Lower & Upper \\
\hline \multicolumn{8}{|c|}{ Slope of reaction times/temporal similarity tested against zero (all trials) } \\
\hline Jupiter & $-0.59(1.04)$ & -3.99 & 49 & $<0.001$ & -0.56 & -0.88 & -0.29 \\
\hline Mars & $-1.15(1.18)$ & -6.86 & 49 & $<0.001$ & -0.97 & -1.48 & -0.81 \\
\hline Saturn & $-0.24(0.69)$ & -2.46 & 49 & 0.020 & -0.35 & -0.43 & -0.04 \\
\hline Mercury & $-0.52(0.73)$ & -5.02 & 49 & $<0.001$ & -0.71 & -0.73 & -0.31 \\
\hline Uranus & $-0.44(0.64)$ & -4.93 & 49 & $<0.001$ & -0.70 & -0.62 & -0.26 \\
\hline Neptune & $-1.03(1.94)$ & -3.65 & 46 & $<0.001$ & -0.53 & -1.60 & -0.46 \\
\hline \multicolumn{8}{|c|}{ Slope of reaction times/temporal similarity tested against zero (correct trials) } \\
\hline Jupiter & $-0.63(1.45)$ & -3.06 & 49 & 0.004 & -0.43 & -1.04 & -0.22 \\
\hline Mars & $-1.01(1.44)$ & -4.98 & 49 & $<0.001$ & -0.70 & -1.42 & -0.60 \\
\hline Saturn & $-0.29(0.80)$ & -2.58 & 49 & 0.013 & -0.36 & -0.52 & -0.06 \\
\hline Mercury & $-0.46(0.89)$ & -3.64 & 49 & $<0.001$ & -0.51 & -0.71 & -0.20 \\
\hline Uranus & $-0.46(0.70)$ & -4.64 & 49 & $<0.001$ & -0.66 & -0.66 & -0.26 \\
\hline Neptune & $-1.01(2.69)$ & -2.56 & 46 & 0.014 & -0.37 & -1.80 & -0.22 \\
\hline \multicolumn{8}{|c|}{ Slope of reaction times/temporal similarity tested against zero (Incorrect trials) } \\
\hline Jupiter & $-0.63(1.40)$ & -3.17 & 49 & 0.003 & -0.45 & -1.02 & -0.23 \\
\hline Mars & $-1.31(1.70)$ & -5.43 & 49 & $<0.001$ & -0.77 & -1.79 & -0.82 \\
\hline Saturn & $-0.25(0.28)$ & -1.39 & 49 & 0.169 & -0.20 & -0.61 & 0.11 \\
\hline Mercury & $-0.55(1.40)$ & -2.81 & 49 & 0.007 & -0.40 & -0.95 & -0.16 \\
\hline Uranus & $-0.42(0.90)$ & -3.29 & 49 & 0.002 & -0.47 & -0.68 & -0.16 \\
\hline Neptune & $-1.27(3.42)$ & -2.55 & 46 & 0.014 & -0.37 & -2.27 & -0.27 \\
\hline
\end{tabular}

885

886

887

888

889 
bioRxiv preprint doi: https://doi.org/10.1101/2020.01.10.902130; this version posted January 11,2020 . The copyright holder for this preprint (which was not certified by peer review) is the author/funder, who has granted bioRxiv a license to display the preprint in perpetuity. It is made available under aCC-BY 4.0 International license.

890 Table S3. For human participants: One sample $t$-tests results of the slopes of RT as a

891 function of temporal similarity (upper panel) and the slopes of RT as a function of

892 chosen frame location (bottom panel) against zero. Related to Figure 1D.

893

894

\begin{tabular}{cccccccc}
\hline Subjects & Mean (SEM) & t-statistics & d.f. & p-value & Cohen's d & $\begin{array}{c}95 \% \text { confidence interval } \\
\text { Lower }\end{array}$ \\
& & & & & & & \\
Upper
\end{tabular}

895

896

897

898

899

900

901

902

903

904

905

906

907

908

909

910 